Rev. Int. Contam. Ambie. 36 (4) 875-892, 2020

https://doi.org/10.20937/RICA.53473

\title{
APORTE CUANTITATIVO DE LAS FUENTES DE PM 10 Y PM 2.5 EN SITIOS URBANOS DEL VALLE DEL MANTARO, PERÚ
}

Apportionment of emission sources of $\mathrm{PM}_{10}$ and $\mathrm{PM}_{2.5}$ At urban sites of mantaro valley, peru

\section{Daniel ÁLVAREZ-TOLENTINO ${ }^{1}$ and Luis SUÁREZ-SALAS ${ }^{2 *}$}

\author{
${ }^{1}$ Universidad Nacional del Centro del Perú, Av. Mariscal Castilla 3909, El Tambo, Huancayo, Perú. \\ ${ }^{2}$ Instituto Geofísico del Perú, Calle Badajoz 169, Urb. Mayorazgo, Ate, Lima, Perú. \\ *Autor para correspondencia: 1suarez@igp.gob.pe
}

(Recibido: noviembre 2018; aceptado: enero 2020)

Palabras clave: partículas atmosféricas, composición química, emisiones, análisis de componentes principales (ACP), Jauja, Concepción, Huancayo.

\section{RESUMEN}

Las partículas atmosféricas $\left(\mathrm{PM}_{10}\right.$ y $\left.\mathrm{PM}_{2.5}\right)$ son responsables de serios problemas en la salud humana. Por ello, este estudio determina el aporte cuantitativo de las fuentes de ambos tipos de partículas en las principales ciudades del Valle del Mantaro, ubicadas en la parte central del Perú. Se analizan los datos obtenidos en la campaña de monitoreo mensual de los centros urbanos de Jauja, Concepción y Huancayo, desde julio de 2007 hasta octubre de 2008. Para el monitoreo se empleó un muestreador de bajo volumen (modelo Partisol). La composición química elemental fue obtenida por fluorescencia de rayos $\mathrm{X}$ y se les aplicó el análisis de componentes principales con rotación varimax y análisis de componentes principales absoluto. Los agrupamientos de los elementos químicos fueron contrastados con el factor de enriquecimiento y análisis de conglomerados jerárquico. Los resultados mostraron que las $\mathrm{PM}_{10}$ y $\mathrm{PM}_{2.5}$ fueron significativamente mayores en Huancayo $(\mathrm{p}<0.05)$, zona urbana donde se superó el Estándar de Calidad Ambiental para Aire (ECA) de la legislación peruana, para ambos tamaños de partículas. Se determinaron trazadores de fuentes naturales y antrópicas. En total se detectaron cinco fuentes de emisión para los sitios urbanos del Valle del Mantaro: polvo del suelo (Al, Ca, Si, Fe, Ti, Mn y K), quema de biomasa (Cl, Br, K), vehículos $(\mathrm{Cu}, \mathrm{Zn}, \mathrm{Cl}, \mathrm{Cr})$, combustible-aceite $(\mathrm{Ni})$ y fundición $(\mathrm{Pb}, \mathrm{Zn}, \mathrm{As}$ y $\mathrm{Cu}$ ), siendo el polvo del suelo la principal fuente de aporte de $\mathrm{PM}_{10}$ y $\mathrm{PM}_{2.5}$.

Key words: particulate matter, chemical composition, emissions, principal component analysis, Jauja, Concepción, Huancayo.

\begin{abstract}
Particulate matter $\left(\mathrm{PM}_{10}\right.$ and $\left.\mathrm{PM}_{2.5}\right)$ is responsible for serious human health problems. Therefore, this study determines the quantitative contribution of the sources of both particles at main cities of Mantaro Valley, located in central Peru. The obtained data in the monthly monitoring campaign of urban sites of Jauja, Concepción and Huancayo, from July 2007 to October 2008 are analyzed. A low-volume sampler (Partisol model) was used for monitoring. Elemental chemical composition was obtained by X-ray
\end{abstract}


fluorescence, and principal component analysis with varimax rotation and absolute principal component analysis were applied. Chemical element groupings were contrasted with enrichment factor and hierarchical cluster analysis. The results showed that $\mathrm{PM}_{10}$ and $\mathrm{PM}_{2.5}$ were significantly higher in Huancayo $(\mathrm{p}<0.05)$, urban area where the National Air Quality Standards for both particle sizes were exceeded. Natural and anthropogenic source tracers were determined. In total, five emission sources were detected for the urban sites of the Mantaro Valley: soil dust (Al, Ca, Si, Fe, Ti, Mn and $\mathrm{K})$, biomass burning $(\mathrm{Cl}, \mathrm{Br}, \mathrm{K})$, vehicles $(\mathrm{Cu}, \mathrm{Zn}, \mathrm{Cl}, \mathrm{Cr})$, fuel-oil ( $\mathrm{Ni}$ ) and smelting $(\mathrm{Pb}, \mathrm{Zn}, \mathrm{As}$ and $\mathrm{Cu})$, being soil dust the main source of $\mathrm{PM}_{10}$ and $\mathrm{PM}_{2.5}$.

\section{INTRODUCCIÓN}

Las partículas atmosféricas (PM, del inglés particulate matter) son una mezcla compleja de pequeñas partículas sólidas y líquidas que se encuentran suspendidas en el aire (Meszaros 1999). Las más preocupantes son las partículas menores de $2.5 \mu \mathrm{m}\left(\mathrm{PM}_{2.5}\right)$ y menores de $10 \mu \mathrm{m}\left(\mathrm{PM}_{10}\right)$ cuya concentración y composición química varían según la ubicación geográfica y condiciones meteorológicas (Pöschl 2005).

Los principales efectos de las PM están asociados al clima y la salud pública. En el clima, causan un efecto directo de enfriamiento del planeta debido a su capacidad para reflejar la radiación solar. Además tienen un efecto indirecto debido a que promueven la formación de los núcleos de condensación de las nubes, incrementando el reflejo de la radiación solar (Andreae y Crutzen 1997, Pöschl 2005, Querol 2008, Olivier y David 2013). En la salud pública, los efectos de la exposición a las PM están fuertemente asociados con el tamaño, la composición y la concentración de las partículas (Davidson et al. 2005), pudiendo provocar enfermedades respiratorias y cardiovasculares (Hamanaka y Mutlu 2018, Hime et al. 2018). La Organización Mundial de la Salud (OMS) estima que 9 de cada 10 personas respiran aire contaminado (OMS 2018) siendo las partículas atmosféricas las responsables del $7.6 \%$ de las muertes mundiales (Cohen et al. 2017). En áreas urbanas, destacan las emisiones vehiculares (Pant y Harrison 2013) siendo responsables, por ejemplo, de 200000 muertes prematuras en los Estados Unidos de América cada año (Caiazzo et al. 2013); asimismo, en Lima (Perú), se ha mostrado la relación de las emisiones del transporte con la frecuencia de asma en niños (Carbajal-Arroyo et al. 2007, Tapia et al. 2018).

Para el diseño del control y la mitigación de la exposición a PM, es importante la identificación y cuantificación del aporte de las fuentes de emisión; para ello, en la identificación se usan trazadores elementales (Chavent et al. 2009) como C/Fe/Ba/Zn/Cu, indicadores de fuentes vehiculares, $\mathrm{Al} / \mathrm{Si} / \mathrm{Ca} / \mathrm{Fe}$ del suelo o la corteza terrestre, $\mathrm{Na} / \mathrm{Cl} / \mathrm{Mg}$ del océano y $\mathrm{V} / \mathrm{Ni}$ /, de emisiones industriales o quemas de combustible, que también están asociados con aerosoles secundarios (Viana et al. 2008). Los elementos $\mathrm{S} / \mathrm{K} /$ $\mathrm{Cl} / \mathrm{Ca} / \mathrm{Br} / \mathrm{Zn}$ son considerados trazadores de quema de biomasa (Echalar et al. 1998, Gilardoni et al. 2011) cobrando importancia en la región tropical que posee una alta frecuencia de quemas e incendios forestales (Reddington et al. 2019).

Uno de los modelos receptores exploratorios recomendados para estudios de calidad del aire urbano (Belis et al. 2014) es el análisis de componentes principales absoluto (ACPA), el cual calcula las puntuaciones absolutas (aportes) de los componentes (fuentes) a través de la regresión de las masas diarias y las concentraciones químicas elementales (Thurston y Spengler 1985). En América del Sur, varios autores, como Artaxo et al. (1999), Echalar et al. (1998), Gidhagen et al. (2002) y Goix et al. (2011) han empleado este modelo con resultados favorables.

En la región tropical, sobre los Andes centrales del Perú, se ubica el Valle del Mantaro (VM), cuyo nombre proviene del principal río que recorre la llanura, dividiéndola en dos márgenes. En este valle se ubican ciudades de importancia económica para la Región Junín y la capital del Perú (Lima) debido a que posee una gran productividad agropecuaria y comercial. Por el incremento poblacional y la diversificación de actividades de origen antrópico, la calidad de aire se ha venido degradando (CONAM 2005, Suárez-Salas et al. 2017) a tal punto que el Ministerio de Ambiente de Perú (MINAM) tiene incluida a la capital comercial del valle (Huancayo) dentro de las 31 zonas con atención prioritaria para la implementación de planes de acción de mejora de la calidad de aire (MINAM 2010). Por ello, se han desarrollado una serie de estudios (Suárez-Salas et al. 2017, Suárez- Salas et al. 2017, Estevan et al. 2019 y Huamán De La Cruz et al. 2019) cuya información necesita ser complementada con la determinación y cuantificación de las fuentes 
de emisión de $\mathrm{PM}_{10}$ y $\mathrm{PM}_{2.5}$ en el VM. Este estudio procesa los datos obtenidos en los años 2007-2008 para brindar información técnica para el desarrollo e implementación de políticas, planes y programas en favor de la mejora de la calidad de aire y protección de la salud de la población.

\section{MATERIALES Y MÉTODOS}

\section{Ubicación de las estaciones de monitoreo}

La presente investigación se enfocó en las áreas urbanas del VM, que consiste en las ciudades de Jauja, Concepción y Huancayo, cuya población total es de aproximadamente 600000 habitantes (INEI 2007). La figura 1 muestra las ubicaciones de las estaciones de monitoreo de $\mathrm{PM}_{10}$ y $\mathrm{PM}_{2.5}$, instaladas en Jauja
(JA, 11.77 S 75.49 W, 3475 msnm), Concepción (CON, 11.9 S $75.31 \mathrm{~W}, 3300 \mathrm{msnm})$ y Huancayo (HYO, 12.07 S, $75.21 \mathrm{~W}, 3272 \mathrm{msnm}$ ), las cuales se encuentran ubicadas sobre la llanura del VM rodeada de montañas desde los 3200 hasta los $4100 \mathrm{msnm}$.

En el VM la actividad económica principal es la comercial seguida del transporte público. Rodeando a las urbes, existen grandes extensiones de terreno con uso agrícola y ganadero cuyas poblaciones se encuentran conectadas por la carretera central, principal vía de la zona.

\section{Climatología}

La figura 2 ilustra los climatogramas obtenidos de las estaciones de Jauja (11.74S 75.47 W) e Ingenio (11.88 S 75.26 W) del Servicio Nacional de Meteorología e Hidrología (SENAMHI) y la estación del
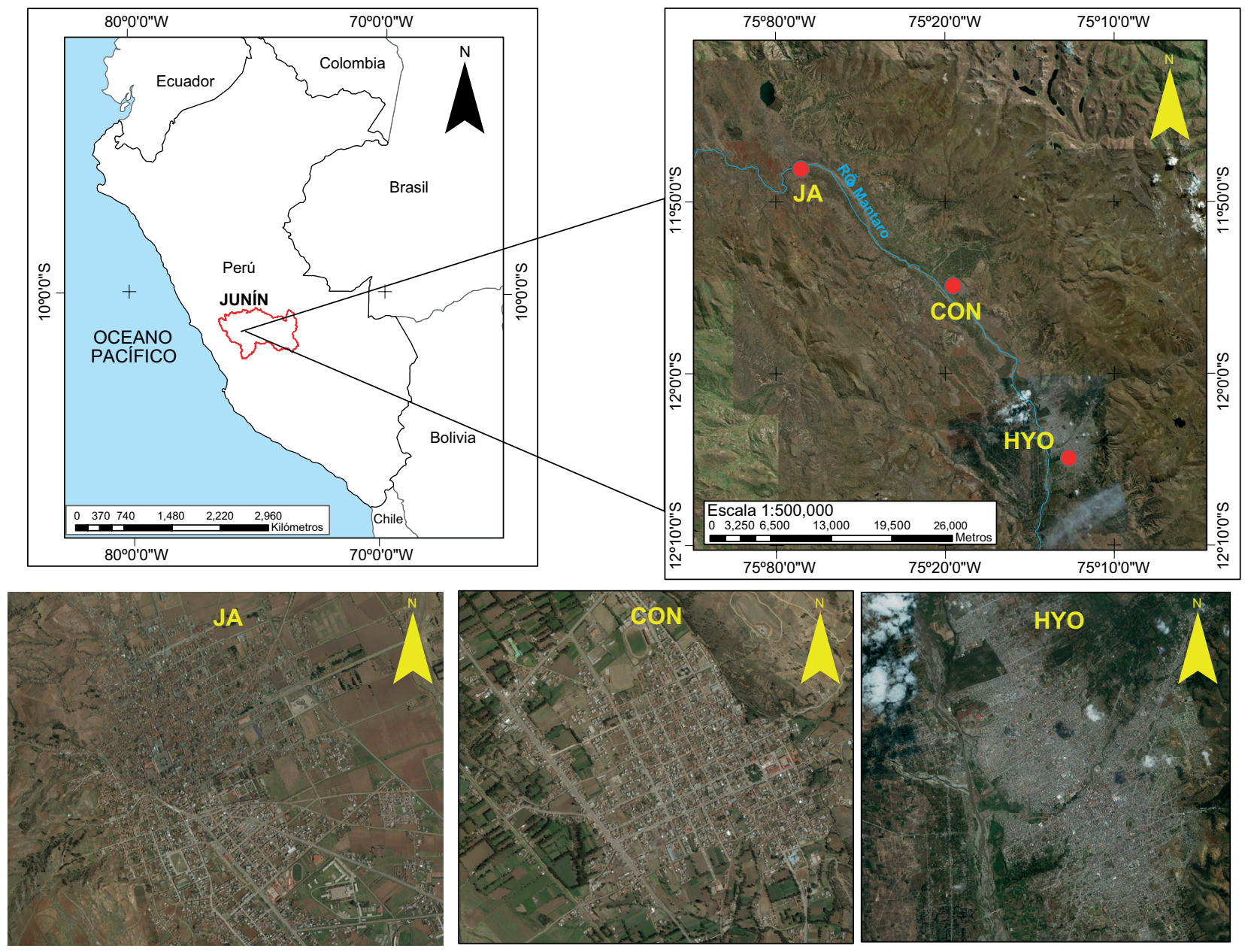

Fig. 1. Ubicación de las estaciones de monitoreo de partículas atmosféricas en los centros urbanos del Valle del Mantaro $(\mathrm{JA}=$ Jauja, $\mathrm{CON}=$ Concepción y HYO = Huancayo), Perú. 


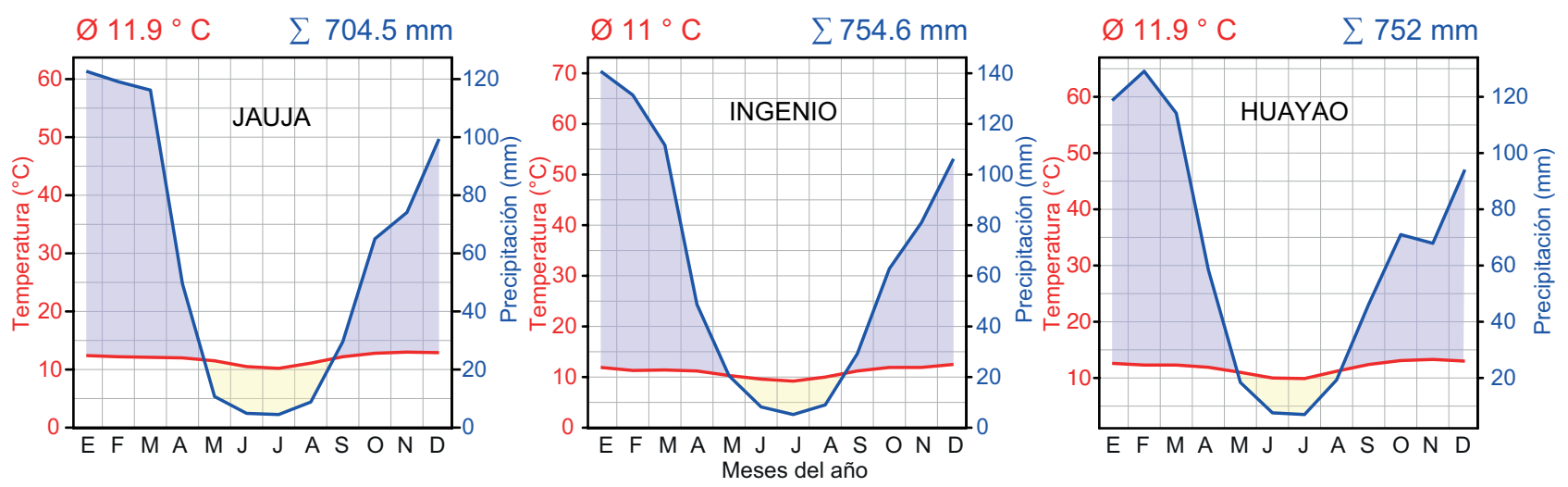

Fig. 2. Climatología de la temperatura media del aire y precipitación promedio del Valle del Mantaro durante los años 1966-2002. Fuente de datos: IGP (2005).

Huayao (12.04 S, 75.32 W) del Instituto Geofísico del Perú (IGP) desde 1966 hasta 2002 (IGP 2005). Estas estaciones son las más cercanas a los sitios de muestreo.

En las tres estaciones la variación de la temperatura media describe dos períodos definidos durante el año, el primero de valores mínimos, centrados en junio y julio (invierno), y el segundo de valores máximos entre octubre y diciembre (primavera-verano). La temperatura media anual para Jauja es de $11.9 \pm$ $0.9{ }^{\circ} \mathrm{C}$, para Ingenio de $11 \pm 1{ }^{\circ} \mathrm{C}$ y para Huayao de $11.9 \pm 1.2^{\circ} \mathrm{C}$.

Del mismo modo, la precipitación en las tres estaciones registra dos períodos bien definidos, el primero corresponde a la época seca (junio-septiembre), y el segundo a la época húmeda (octubre-mayo), siendo enero (para la estaciones JA y CON) y febrero (para HYO) los meses de máxima precipitación. El promedio anual de precipitación para JA fue de $704 \pm$ $47.2 \mathrm{~mm}$, para CON de $755 \pm 50.2 \mathrm{~mm}$ y para $\mathrm{HYO}$ de $752 \pm 44.3 \mathrm{~mm}$.

\section{Muestreo de partículas atmosféricas}

La campaña de monitoreo de $\mathrm{PM}_{10}$ y $\mathrm{PM}_{2.5}$ se llevó a cabo con un muestreador de bajo volumen (Partisol FRM 2000 de Thermo Scientific, EUA) a 16.7 L/min por un tiempo de 24 horas. Se empleó un ciclón para la colecta de $\mathrm{PM}_{2.5} \mathrm{y}$ un cabezal de tipo inlet para $\mathrm{PM}_{10}$ (USEPA). En cada estación, el equipo se instaló entre los 8 y $10 \mathrm{~m}$ del suelo, cerca de las intersecciones de calles de gran confluencia vehicular y comercial; complementariamente se instaló una estación meteorológica (modelo 232 de Weatherhawk, EUA).

La campaña de monitoreo se inició el 31 de julio de 2007 y terminó el 17 de octubre de 2008; durante este tiempo se realizaron colectas de $\mathrm{PM}_{10}$, con una frecuencia de monitoreo de cada tres días, y colectas de $\mathrm{PM}_{2.5}$, con una frecuencia de monitoreo de cada 15 días. Ambos tamaños de partículas fueron colectados en filtros de teflón con diámetro de $47 \mathrm{~mm}$ y tamaño de poro de $2.0 \mu \mathrm{m}$.

La colecta de $\mathrm{PM}_{10}$ y $\mathrm{PM}_{2.5}$ se desarrolló en forma secuencial, por ello, los equipos de monitoreo se trasladaron de una estación a otra, cada mes. El detalle de los meses de monitoreo y la cantidad de filtros colectados en cada estación del VM se describen en el cuadro I. Para el aseguramiento de la calidad del proceso de monitoreo se emplearon filtros testigo (10\% de toda la campaña), cuyos resultados en su mayoría fueron menores al límite de detección, a excepción de los elementos: $\mathrm{Ca}, \mathrm{Cr}, \mathrm{Ge}, \mathrm{Fe}, \mathrm{V}, \mathrm{Co}, \mathrm{Cu}$ y Ni, Ti y $\mathrm{Zn}$ para filtros testigo de $\mathrm{PM}_{10}$ y $\mathrm{Co}, \mathrm{Cu}, \mathrm{Ge}, \mathrm{Fe}$, $\mathrm{Mn}, \mathrm{Ni}$ y $\mathrm{Zn}$ en filtros testigo de $\mathrm{PM}_{2.5}$. Los valores de los elementos detectados en los filtros en testigo fueron restados a los resultados de las muestras.

\section{Análisis químico de las muestras}

Los filtros obtenidos en toda la campaña fueron analizados por el Laboratorio Chester LabNet en EUA, certificado por el Programa Nacional de Acreditación de Laboratorios Ambientales (NELAC, por sus siglas en inglés). La masa se determinó por el método gravimétrico (EPA IO-3.1) mediante una microbalanza de $\pm 0.01 \mathrm{mg}$ de precisión. Las concentraciones de los elementos químicos se determinaron mediante la técnica de fluorescencia de rayos $\mathrm{X}(\mathrm{XRF})$ establecida en el método analítico EPA IO-3.3. Este método consiste en bombardear con rayos $\mathrm{X}$ a los filtros con muestra, para que, con base en su excitación y liberación de energía, se determine su concentración según la huella digital 
CUADRO I. DETALLE DEL MONITOREO DE PARTÍCULAS ATMOSFÉRICAS REALIZADO EN LOS CENTROS URBANOS DEL VALLE DEL MANTARO.

\begin{tabular}{lllcc}
\hline Estación & Descripción & Meses de monitoreo & $\begin{array}{c}\text { Número } \\
\text { muestras PM } 10\end{array}$ & $\begin{array}{c}\text { Número } \\
\text { muestras PM }_{2.5}\end{array}$ \\
\hline $\begin{array}{l}\text { Jauja } \\
\text { (JA) }\end{array}$ & $\begin{array}{l}\text { Ubicada en la azotea de un hos- } \\
\text { pedaje a 10 m de altura, a } \\
5 \text { cuadras de la plaza principal. }\end{array}$ & $\begin{array}{l}\text { septiembre, noviembre (2007). } \\
\text { febrero, junio (2008). }\end{array}$ & 41 & 13 \\
\hline $\begin{array}{l}\text { Concepción } \\
\text { (CON) }\end{array}$ & $\begin{array}{l}\text { Ubicada en el auditorio munici- } \\
\text { pal a 8 m de altura, al frente de la } \\
\text { plaza principal. }\end{array}$ & $\begin{array}{l}\text { octubre, diciembre (2007). } \\
\text { marzo, julio, agosto, septiembre, } \\
\text { octubre (2008). }\end{array}$ & 47 & 11 \\
\hline $\begin{array}{l}\text { Huancayo } \\
\text { (HYO) }\end{array}$ & $\begin{array}{l}\text { Ubicada en el Colegio Rosario } \\
\text { a } 8 \text { m de altura, a 2 cuadras de } \\
\text { la plaza principal. }\end{array}$ & $\begin{array}{l}\text { agosto (2007). } \\
\text { enero, abril, mayo (2008) }\end{array}$ & 41 & 9 \\
\hline
\end{tabular}

de cada elemento. Los límites de detección de los 38 elementos analizados por XRF se muestran en el cuadro II.

CUADRO II. LÍMITES DE DETECCIÓN DE LOS ELEMENTOS QUÍMICOSANALIZADOS EN LAS PARTÍCULAS ATMOSFÉRICAS.

\begin{tabular}{cccccc}
\hline Elemento & $\mu \mathrm{g} / \mathrm{m}^{3}$ & Elemento & $\mu \mathrm{g} / \mathrm{m}^{3}$ & Elemento & $\mu \mathrm{g} / \mathrm{m}^{3}$ \\
\hline $\mathrm{Al}$ & 0.008 & $\mathrm{Sr}$ & 0.0022 & $\mathrm{Ag}$ & 0.0075 \\
$\mathrm{Sb}$ & 0.011 & $\mathrm{P}$ & 0.0047 & $\mathrm{~Pb}$ & 0.0039 \\
$\mathrm{As}$ & 0.002 & $\mathrm{Ga}$ & 0.0021 & $\mathrm{~K}$ & 0.0030 \\
$\mathrm{~S}$ & 0.004 & $\mathrm{Ge}$ & 0.0013 & $\mathrm{Rb}$ & 0.0014 \\
$\mathrm{Ba}$ & 0.083 & $\mathrm{Fe}$ & 0.0008 & $\mathrm{Se}$ & 0.0014 \\
$\mathrm{Br}$ & 0.001 & $\mathrm{In}$ & 0.0196 & $\mathrm{Si}$ & 0.0055 \\
$\mathrm{Cd}$ & 0.008 & $\mathrm{La}$ & 0.0508 & $\mathrm{Na}$ & 0.1484 \\
$\mathrm{Ca}$ & 0.002 & $\mathrm{Mg}$ & 0.0178 & $\mathrm{Ti}$ & 0.0014 \\
$\mathrm{Cl}$ & 0.005 & $\mathrm{Mn}$ & 0.0015 & $\mathrm{~V}$ & 0.0010 \\
$\mathrm{Co}$ & 0.001 & $\mathrm{Hg}$ & 0.0035 & $\mathrm{Y}$ & 0.0021 \\
$\mathrm{Cu}$ & 0.001 & $\mathrm{Mo}$ & 0.0035 & $\mathrm{Zn}$ & 0.0010 \\
$\mathrm{Cr}$ & 0.001 & $\mathrm{Ni}$ & 0.0008 & $\mathrm{Zr}$ & 0.0025 \\
$\mathrm{Sn}$ & 0.013 & $\mathrm{Pd}$ & 0.0071 & & \\
\hline
\end{tabular}

\section{Análisis estadístico}

Los resultados del laboratorio ( $\mu \mathrm{g} /$ filtro) fueron convertidos a $\mu \mathrm{g} / \mathrm{m}^{3}$ por el método EPA IO-2.4, tomando en cuenta el flujo de aire que circuló durante las $24 \mathrm{~h}$ de muestreo, la temperatura y la presión atmosférica de cada estación de monitoreo.

Se calcularon los estadísticos descriptivos y pruebas de $\mathrm{T}$, análisis de varianza y Kruskal-Wallis con nivel de significancia del $95 \%(p<0.05)$. Se compararon las concentraciones de $\mathrm{PM}_{10}$ y $\mathrm{PM}_{2.5}$ con los Estándares de Calidad Ambiental (ECA) establecidos por la normatividad peruana (MINAM 2017).

\section{Factor de enriquecimiento}

Para diferenciar a los elementos químicos procedentes de actividades de origen en fuentes naturales y antrópicas, se aplicó el factor de enriquecimiento (FE). Para ello se usó la concentración de referencia del aluminio (Al) de Wedepohl K H. (1995), bajo la siguiente ecuación (Rahn 1971):

$\mathrm{FE}=(\mathrm{X} / \mathrm{Al})_{(\text {aire })} /(\mathrm{X} / \mathrm{Al})_{(\text {corteza terrestre })}$

Donde $\mathrm{X}$ es la concentración de cada elemento obtenida en cada muestra, Al es la concentración de referencia del Al. Cuando el FE de una muestra se aproxima a la unidad, la fuente dominante para ese elemento es la corteza terrestre; Si FE > 10, una fracción significativa del elemento es de una fuente de origen antrópico (Chester et al. 2000).

\section{Análisis multivariado}

Se aplicó el análisis de componentes principales (ACP) con método varimax a las concentraciones elementales estandarizadas a media cero y varianza unidad (Pison et al. 2003). Para el caso de las $\mathrm{PM}_{10}$ de cada estación, se consideró a los elementos químicos cuyos datos fueron mayores a 30 (Hopke 2010); para las $\mathrm{PM}_{2.5}$, dado el menor número de muestras, se consideró a los elementos químicos trazadores, cuidando que el número de datos fuera mayor al número de elementos (Husson et al. 2010). El número de componentes (factores) considerados para el análisis e interpretación, en cada estación, fueron los factores con autovalores mayores a 1 (Kaiser 1958). Los elementos químicos con cargas factoriales mayores a 0.5 fueron considerados influyentes para cada componente.

Para confirmar los factores seleccionados, se utilizó el análisis de conglomerado jerárquico (ACJ) 
con método Ward y distancia euclidiana al cuadrado para identificar similitudes y características de las asociaciones entre los elementos químicos. El número de grupos se realizó conforme al número de factores determinados.

Dado que el ACP proporciona un reparto de los elementos químicos por factores, cada factor fue considerado como una fuente de emisión de PM. Para estimar la contribución de cada fuente se realizó el análisis de componentes principales absoluto (ACPA) según la metodología de Thurston y Spengler (1985).

En todos los análisis realizados se empleó el programa libre R versión 3.5.1 (R Core Team 2015).

\section{RESULTADOS Y DISCUSIÓN}

\section{Dirección y velocidad de los vientos}

Como se observa en la figura 3, en los meses monitoreados de la estación de JA, los vientos predominantes provienen del nornoroeste y norte, con velocidades dominantes comprendidas entre 7 a $31.3 \mathrm{~m} / \mathrm{s}$; asimismo, en la estación de CON, los vientos predominantes provinieron del noreste con velocidades comprendidas entre 1 a $3 \mathrm{~m} / \mathrm{s}$; en el caso de la estación de HYO, los vientos predominantes provinieron del sursuroeste y sur con velocidades dominantes de 1 a $3 \mathrm{~m} / \mathrm{s}$. En las tres estaciones, se observan vientos provenientes de otras direcciones, lo cual indica la existencia de mezcla de masas de aire (Flores-Rojas et al. 2019).

\section{Partículas atmosféricas PM10}

En el cuadro III, se muestran las concentraciones promedio \pm desviación estándar (prom $\pm \mathrm{ds}$ ), valor máximo (máx.) y cantidad de datos (N) de las $\mathrm{PM}_{10} \mathrm{y}$ los elementos químicos de las estaciones JA, CONy HYO. La concentración de $\mathrm{PM}_{10}$ en el centro urbano de Huancayo $\left(65 \pm 31 \mu \mathrm{g} / \mathrm{m}^{3}\right)$ fue significativamente mayor $(\mathrm{F}=44.25 ; \mathrm{p}<0.0001)$ con respecto a las demás estaciones. Por lo tanto, el área urbana de Huancayo posee la mayor problemática de partículas del VM. Huancayo es considerada la capital comercial y económica de la región Junín y alberga el 84 \% de la población del VM (aproximadamente 500000 habitantes). No se encontró diferencia significativa $(\mathrm{T}=0.09 ; \mathrm{p}=0.93)$ entre las $\mathrm{PM}_{10}$ de JA $(32 \pm$ $\left.12 \mu \mathrm{g} / \mathrm{m}^{3}\right)$ y CON $\left(28 \pm 13 \mu \mathrm{g} / \mathrm{m}^{3}\right)$, lo cual se debe a que son ciudades más pequeñas.

Los tres elementos químicos con mayor concentración registrada en las $\mathrm{PM}_{10}$ en las tres estaciones de monitoreo fueron $\mathrm{Si}$, Ca y Al, trazadores de fuentes geológicas o suelo. Este resultado es razonable por la mezcla de vientos que transporta materiales de áreas rurales y calles que no están pavimentados, por otro lado, en el tiempo de monitoreo se observaron diversas construcciones de edificios.
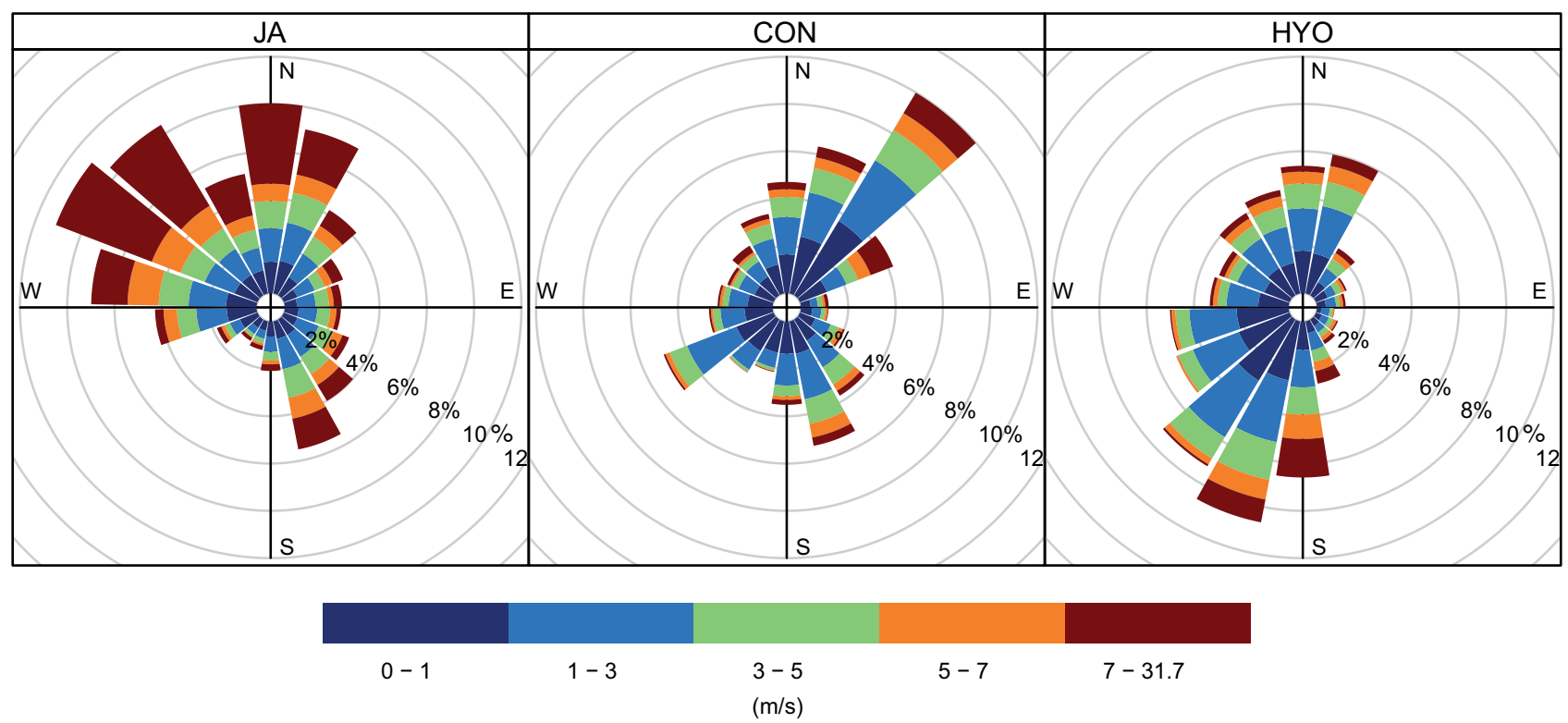

Fig. 3. Rosas de vientos obtenidas en las estaciones de monitoreo de Valle del Mantaro $(\mathrm{JA}=\mathrm{Jauja}$, CON $=\mathrm{Concepción} \mathrm{y} \mathrm{HYO}=$ Huancayo), Perú. Las barras indican la dirección de procedencia y los porcentajes su frecuencia. 
CUADRO III. CONCENTRACIÓN PROMEDIO ( $($ DESVIACIÓN ESTÁNDAR) DE LAS PARTÍCULAS ATMOSFÉRICAS $\left(\mathrm{PM}_{10}\right)$ Y COMPOSICIÓN QUÍMICA ELEMENTAL, EXPRESADA EN $\mu \mathrm{g} / \mathrm{m}^{3}$, EN LOS CENTROS URBANOS DEL VALLE DEL MANTARO, PERÚ.

\begin{tabular}{|c|c|c|c|c|c|c|c|c|c|c|c|c|c|c|c|}
\hline \multicolumn{6}{|c|}{$\mathrm{JA}$} & \multicolumn{5}{|c|}{$\mathrm{CON}$} & \multicolumn{5}{|c|}{ HYO } \\
\hline & Pro & $\mathrm{m} \pm \mathrm{c}$ & & Máx & $\mathrm{N}$ & & Pro & $\mathrm{m} \pm \mathrm{de}$ & Máx & $\mathrm{N}$ & & Pro1 & $\mathrm{m} \pm \mathrm{de}$ & máx & $\mathrm{N}$ \\
\hline $\mathrm{PM}_{10}$ & 31.7 & \pm 11 & & 52.9 & 41 & $\mathrm{PM}_{10}$ & 28.2 & \pm 13.1 & 71.3 & 47 & $\mathrm{PM}_{10}$ & 64.5 & \pm 30.9 & 143 & 41 \\
\hline $\mathrm{Si}$ & 1.8 & \pm 0 & & 3.2 & 41 & $\mathrm{Si}$ & 1.8 & \pm 0.9 & 3.7 & 47 & $\mathrm{Si}$ & 4.8 & \pm 2.4 & 11 & 40 \\
\hline $\mathrm{Ca}$ & 1.3 & \pm 0 & & 2.2 & 41 & $\mathrm{Al}$ & 0.9 & \pm 0.6 & 3.9 & 45 & $\mathrm{Ca}$ & 2.4 & \pm 1.2 & 5.8 & 41 \\
\hline $\mathrm{S}$ & 1 & \pm 0 & & 3 & 41 & $\mathrm{Ca}$ & 0.7 & \pm 0.3 & 1.5 & 47 & $\mathrm{Al}$ & 2 & \pm 1.1 & 4.7 & 40 \\
\hline $\mathrm{Al}$ & 0.6 & \pm 0 & & 1.2 & 41 & $\mathrm{~S}$ & 0.7 & \pm 0.6 & 2.6 & 45 & $\mathrm{Fe}$ & 1.4 & \pm 0.7 & 3.2 & 40 \\
\hline $\mathrm{Fe}$ & 0.6 & \pm 0 & & 1 & 41 & $\mathrm{~K}$ & 0.6 & \pm 0.8 & 5.4 & 46 & $\mathrm{~K}$ & 1.1 & \pm 0.6 & 3.1 & 40 \\
\hline K & 0.5 & \pm 0 & & 0.8 & 41 & $\mathrm{Fe}$ & 0.6 & \pm 0.2 & 1.1 & 45 & $\mathrm{~S}$ & 0.9 & \pm 0.6 & 2.2 & 40 \\
\hline $\mathrm{Na}$ & 0.2 & \pm 0 & .05 & 0.3 & 6 & $\mathrm{Cl}$ & 0.3 & \pm 1.4 & 9.5 & 45 & $\mathrm{Cl}$ & 0.3 & \pm 0.29 & 1.3 & 40 \\
\hline $\mathrm{Pb}$ & 0.2 & \pm 0 & & 0.8 & 40 & $\mathrm{Na}$ & 0.3 & \pm 0.03 & 0.3 & 2 & $\mathrm{Na}$ & 0.2 & \pm 0.1 & 0.4 & 9 \\
\hline As & 0.1 & \pm 0 & & 0.6 & 40 & $\mathrm{Mg}$ & 0.1 & \pm 0.05 & 0.2 & 21 & $\mathrm{Mg}$ & 0.2 & \pm 0.1 & 0.5 & 38 \\
\hline $\mathrm{Cl}$ & 0.1 & \pm 0 & & 0.3 & 37 & $\mathrm{Ti}$ & 0.1 & \pm 0.02 & 0.1 & 46 & $\mathrm{Ti}^{\circ}$ & 0.1 & \pm 0.1 & 0.3 & 40 \\
\hline $\mathrm{Sb}$ & 0.1 & \pm 0 & & 0.4 & 18 & $\mathrm{Zn}$ & 0.03 & \pm 0.01 & 0.1 & 47 & $\mathrm{Zn}$ & 0.1 & \pm 0.03 & 0.1 & 40 \\
\hline $\mathrm{Zn}$ & 0.1 & \pm 0 & & 0.2 & 41 & $\mathrm{~Pb}$ & 0.02 & \pm 0.02 & 0.09 & 43 & $\mathrm{Mn}$ & 0.04 & \pm 0.02 & 0.1 & 40 \\
\hline $\mathrm{Ti}$ & 0.05 & \pm 0 & .02 & 0.08 & 41 & $\mathrm{Sb}$ & 0.02 & \pm 0.004 & 0.02 & 5 & $\mathrm{~Pb}$ & 0.03 & \pm 0.03 & 0.19 & 40 \\
\hline $\mathrm{Mg}$ & 0.04 & \pm 0 & .01 & 0.06 & 14 & $\mathrm{Mn}$ & 0.02 & \pm 0.01 & 0.03 & 45 & As & 0.02 & \pm 0.03 & 0.12 & 38 \\
\hline $\mathrm{Cu}$ & 0.02 & \pm 0 & .02 & 0.08 & 40 & As & 0.01 & \pm 0.01 & 0.08 & 42 & $\mathrm{Sb}$ & 0.02 & \pm 0.005 & 0.03 & 9 \\
\hline Mn & 0.01 & \pm 0 & .01 & 0.03 & 41 & $\mathrm{P}$ & 0.01 & \pm 0.004 & 0.02 & 17 & $\mathrm{P}$ & 0.02 & \pm 0.01 & 0.05 & 25 \\
\hline $\mathrm{Cd}$ & 0.01 & \pm 0 & .003 & 0.02 & 7 & $\mathrm{Sr}$ & 0.01 & \pm 0.02 & 0.1 & 31 & $\mathrm{Cu}$ & 0.01 & \pm 0.01 & 0.02 & 40 \\
\hline $\mathrm{P}$ & 0.01 & \pm 0 & .01 & 0.02 & 5 & $\mathrm{Br}$ & 0.004 & \pm 0.008 & 0.047 & 32 & $\mathrm{Cd}$ & 0.01 & \pm 0.0004 & 0.01 & 3 \\
\hline $\mathrm{Ag}$ & 0.01 & \pm 0 & .001 & 0.01 & 2 & $\mathrm{Cu}$ & 0.004 & \pm 0.002 & 0.011 & 45 & $\mathrm{Sr}$ & 0.01 & \pm 0.004 & 0.02 & 39 \\
\hline $\mathrm{Se}$ & 0.01 & \pm 0 & .002 & 0.01 & 12 & $\mathrm{Zr}$ & 0.003 & \pm 0.001 & 0.005 & 7 & $\mathrm{Zr}$ & 0.005 & \pm 0.002 & 0.01 & 26 \\
\hline $\mathrm{Sr}$ & 0.004 & \pm 0 & .001 & 0.007 & 26 & $\mathrm{Rb}$ & 0.002 & \pm 0.001 & 0.005 & 39 & $\mathrm{~V}$ & 0.005 & \pm 0.003 & 0.012 & 16 \\
\hline $\mathrm{Zr}$ & 0.004 & \pm 0 & .001 & 0.005 & 6 & V & 0.002 & \pm 0.001 & 0.004 & 14 & $\mathrm{Rb}$ & 0.004 & \pm 0.002 & 0.009 & 39 \\
\hline $\mathrm{Rb}$ & 0.003 & \pm 0 & .001 & 0.003 & 19 & $\mathrm{Cr}$ & 0.002 & \pm 0.0004 & 0.002 & 9 & $\mathrm{Br}$ & 0.003 & \pm 0.002 & 0.01 & 35 \\
\hline $\mathrm{Br}$ & 0.002 & \pm 0 & .001 & 0.005 & 14 & $\mathrm{Ge}$ & 0.001 & \pm 0.0004 & 0.001 & 3 & $\mathrm{Cr}$ & 0.003 & \pm 0.002 & 0.008 & 32 \\
\hline $\mathrm{V}$ & 0.002 & \pm 0 & .001 & 0.004 & 10 & $\mathrm{Ni}$ & 0.001 & \pm 0.001 & 0.002 & 22 & $\mathrm{Ni}$ & 0.002 & \pm 0.002 & 0.008 & 15 \\
\hline $\mathrm{Cr}$ & 0.002 & \pm 0 & .001 & 0.003 & 11 & Co & 0.0003 & $3 \pm 0.0004$ & 0.0007 & 3 & $\mathrm{Se}$ & 0.002 & \pm 0.0001 & 0.002 & 2 \\
\hline $\mathrm{Ni}$ & 0.001 & \pm 0 & .001 & 0.004 & 18 & & & & & & & & & & \\
\hline
\end{tabular}

Prom $=$ promedio aritmético, de $=$ desviación estándar, máx $=$ valor máximo, $\mathrm{N}=$ número de datos, $\mathrm{JA}=\mathrm{Jauja}, \mathrm{CON}=\mathrm{Concepción}$, $\mathrm{HYO}=$ Huancayo.

En la figura 4, se observa la variabilidad diaria de las $\mathrm{PM}_{10}$ en las estaciones de JA, CON y HYO, adicionalmente se muestra en línea roja horizontal el ECA peruano $\left(100 \mu \mathrm{g} / \mathrm{m}^{3}\right)$, el cual es el doble del valor recomendado por la OMS $\left(50 \mu \mathrm{g} / \mathrm{m}^{3}\right)(\mathrm{OMS}$ 2006). Sin embargo, en tres días de monitoreo en el centro de HYO fue superado el ECA, correspondiendo estos valores a los días 09/08/2007, 24/08/2007 y $23 / 05 / 2008$. Estos valores altos son un indicador inicial de que los valores de PM representarían un riesgo para la población.

\section{$P M_{2.5}$}

Al igual que en el caso de las $\mathrm{PM}_{10}$, la concentración de las partículas finas del centro urbano de HYO $\left(34 \pm 15 \mu \mathrm{g} / \mathrm{m}^{3}\right)$ fue mayor a lo registrado en las demás estaciones (Cuadro IV); igualmente, el promedio de $\mathrm{PM}_{2.5}$ de JA $\left(22 \pm 14 \mu \mathrm{g} / \mathrm{m}^{3}\right)$ fue superior al de $\operatorname{CON}\left(16 \pm 8 \mu \mathrm{g} / \mathrm{m}^{3}\right)$ existiendo una diferencia significativa entre las concentraciones registradas en las estaciones (Kruskal-Wallis $=8.6 ; p=0.01)$. Los elementos químicos más abundantes en las $\mathrm{PM}_{2.5}$ de las tres estaciones fueron el S, Si y K. Asimismo, se observa mayor detección de elementos químicos en JA (21 elementos químicos) comparado con CON (18 elementos químicos) y HYO (16 elementos).

En la figura 5, se observa la variabilidad diaria de $\mathrm{PM}_{2.5}$ en las estaciones de JA, CON y HYO durante toda la campaña de monitoreo. Comparando los datos, con el ECA peruano de 24 horas $\left(50 \mu \mathrm{g} / \mathrm{m}^{3}\right)$, se comprueba que la norma se rebasó en dos ocasiones: una en HYO $\left(67.8 \mu \mathrm{g} / \mathrm{m}^{3}, 25 / 08 / 2007\right)$ y la otra en JA $\left(51.7 \mu \mathrm{g} / \mathrm{m}^{3}, 02 / 10 / 2007\right)$, las cuales han sido registradas en temporada seca, época donde 


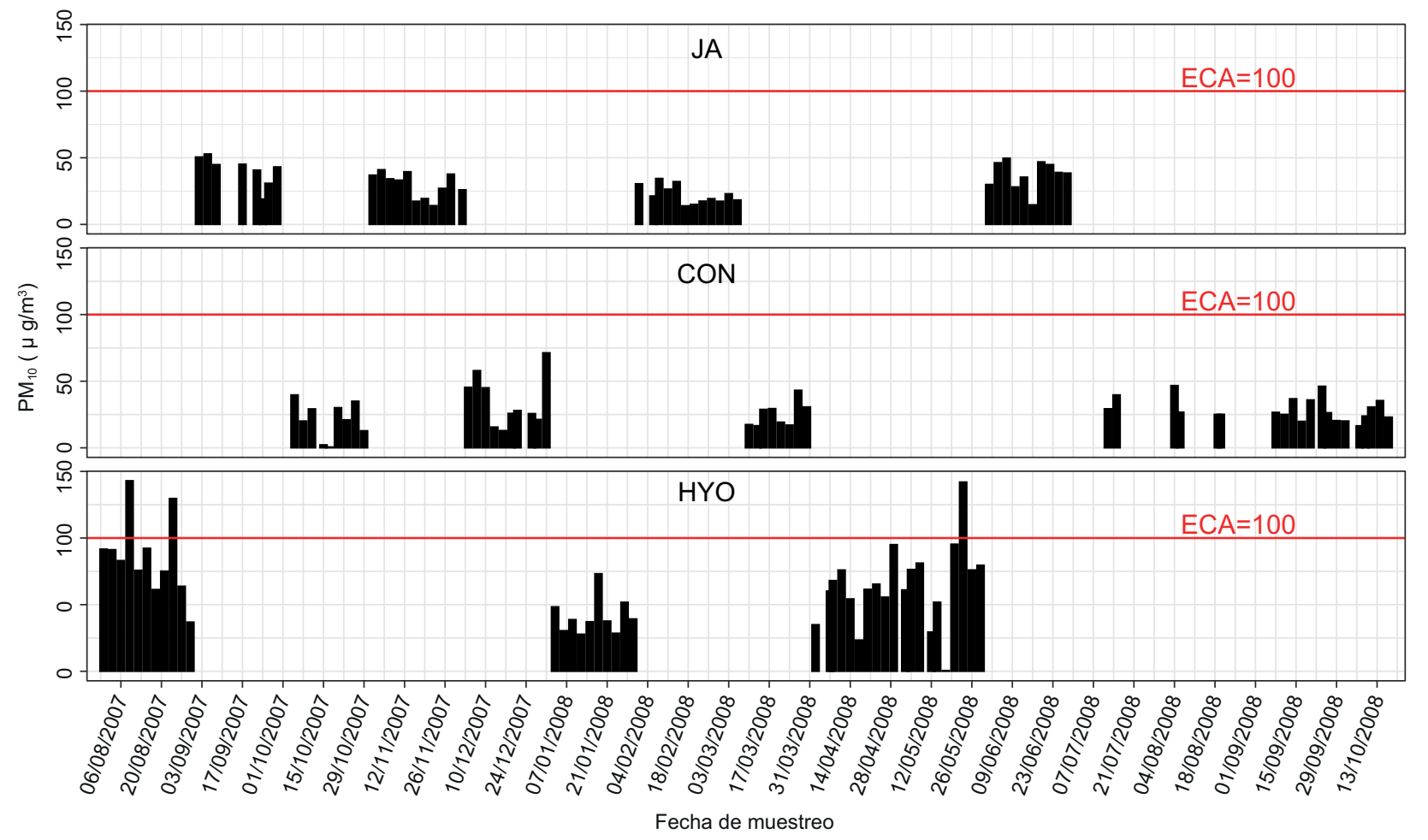

Fig. 4. Variación diaria de las partículas atmosféricas $\left(\mathrm{PM}_{10}\right)$ registradas en los centros urbanos del Valle del Mantaro (JA = Jauja, $\mathrm{CON}=$ Concepción y HYO = Huancayo), Perú. En la línea horizontal roja se muestra el Estándar de Calidad Ambiental peruano de $24 \mathrm{~h}\left(\mathrm{ECA}=100 \mu \mathrm{g} / \mathrm{m}^{3}\right)$.

CUADRO IV. CONCENTRACIÓN PROMEDIO ( \pm DESVIACIÓN ESTÁNDAR) DE LAS PARTÍCULAS ATMOSFÉRICAS PM 2.5 Y COMPOSICIÓN QUÍMICA ELEMENTAL, EXPRESADA EN $\mu \mathrm{g} / \mathrm{m}^{3}$, EN EL VALLE DEL MANTARO, PERÚ.

\begin{tabular}{|c|c|c|c|c|c|c|c|c|c|c|c|c|c|c|}
\hline \multicolumn{5}{|c|}{ JA } & \multicolumn{5}{|c|}{$\mathrm{CON}$} & \multicolumn{5}{|c|}{ HYO } \\
\hline & Pro & $\mathrm{m} \pm \mathrm{de}$ & Máx & $\mathrm{N}$ & & Pro & $\mathrm{m} \pm \mathrm{de}$ & Máx & $\mathrm{N}$ & & Pro & $\mathrm{m} \pm \mathrm{de}$ & Máx & $\mathrm{N}$ \\
\hline $\mathrm{PM}_{2.5}$ & 21.8 & \pm 13.8 & 51.6 & 13 & $\mathrm{PM}_{2.5}$ & 15.8 & \pm 7.8 & 26.6 & 11 & $\mathrm{PM}_{2.5}$ & 34.5 & \pm 14.7 & 67.8 & 9 \\
\hline S & 1.2 & \pm 0.8 & 2.3 & 13 & $\mathrm{~S}$ & 0.9 & \pm 0.9 & 2.4 & 11 & $\mathrm{~S}$ & 0.9 & \pm 0.7 & 1.9 & 9 \\
\hline $\mathrm{Si}$ & 0.4 & \pm 0.3 & 0.9 & 13 & $\mathrm{Si}$ & 0.3 & \pm 0.2 & 0.5 & 11 & $\mathrm{Si}$ & 0.7 & \pm 0.3 & 1.1 & 9 \\
\hline $\mathrm{K}$ & 0.3 & \pm 0.2 & 0.8 & 13 & K & 0.2 & \pm 0.1 & 0.3 & 11 & K & 0.4 & \pm 0.3 & 1 & 9 \\
\hline $\mathrm{Ca}$ & 0.2 & \pm 0.1 & 0.4 & 13 & $\mathrm{Al}$ & 0.2 & \pm 0.1 & 0.3 & 11 & $\mathrm{Ca}$ & 0.3 & \pm 0.1 & 0.5 & 9 \\
\hline $\mathrm{Na}$ & 0.2 & \pm 0.05 & 0.2 & 2 & $\mathrm{Ca}$ & 0.1 & \pm 0.1 & 0.3 & 11 & $\mathrm{Cl}$ & 0.3 & \pm 0.2 & 0.5 & 8 \\
\hline $\mathrm{Al}$ & 0.2 & \pm 0.1 & 0.4 & 13 & $\mathrm{Fe}$ & 0.1 & \pm 0.04 & 0.2 & 11 & $\mathrm{Al}$ & 0.3 & \pm 0.2 & 0.6 & 9 \\
\hline $\mathrm{Cl}$ & 0.1 & \pm 0.1 & 0.3 & 6 & $\mathrm{~Pb}$ & 0.1 & \pm 0.05 & 0.1 & 6 & $\mathrm{Fe}$ & 0.2 & \pm 0.1 & 0.3 & 9 \\
\hline $\mathrm{Pb}$ & 0.1 & \pm 0.2 & 0.7 & 12 & $\mathrm{Cl}$ & 0.1 & \pm 0.1 & 0.2 & 7 & $\mathrm{Mg}$ & 0.04 & \pm 0.01 & 0.05 & 4 \\
\hline $\mathrm{Fe}$ & 0.1 & \pm 0.1 & 0.3 & 13 & $\mathrm{Sb}$ & 0.02 & \pm 0.01 & 0.03 & 2 & $\mathrm{Zn}$ & 0.02 & \pm 0.01 & 0.03 & 9 \\
\hline As & 0.1 & \pm 0.1 & 0.3 & 12 & As & 0.02 & \pm 0.02 & 0.07 & 11 & $\mathrm{~Pb}$ & 0.02 & \pm 0.01 & 0.04 & 9 \\
\hline $\mathrm{Sb}$ & 0.04 & \pm 0.03 & 0.1 & 5 & $\mathrm{Zn}$ & 0.02 & \pm 0.01 & 0.04 & 11 & $\mathrm{Ti}$ & 0.02 & \pm 0.01 & 0.03 & 9 \\
\hline $\mathrm{Mg}$ & 0.04 & \pm 0.02 & 0.1 & 3 & $\mathrm{Ti}$ & 0.01 & \pm 0.01 & 0.02 & 11 & As & 0.01 & \pm 0.01 & 0.03 & 9 \\
\hline $\mathrm{Zn}$ & 0.02 & \pm 0.03 & 0.1 & 13 & $\mathrm{Se}$ & 0.004 & \pm 0.001 & 0.005 & 3 & $\mathrm{Mn}$ & 0.01 & \pm 0.002 & 0.01 & 9 \\
\hline $\mathrm{Cd}$ & 0.01 & \pm 0.005 & 0.02 & 2 & $\mathrm{Mn}$ & 0.004 & \pm 0.001 & 0.01 & 9 & $\mathrm{Cu}$ & 0.00 & $t \pm 0.005$ & 0.01 & 8 \\
\hline $\mathrm{Ti}$ & 0.01 & \pm 0.01 & 0.02 & 13 & $\mathrm{Cu}$ & 0.003 & \pm 0.002 & 0.01 & 8 & $\mathrm{Br}$ & 0.00 & \pm 0.001 & 0.01 & 8 \\
\hline $\mathrm{Cu}$ & 0.01 & \pm 0.01 & 0.02 & 11 & $\mathrm{Ni}$ & 0.003 & \pm 0.001 & 0.004 & 4 & $\mathrm{Ni}$ & 0.00 & \pm 0.002 & 0.004 & 2 \\
\hline $\mathrm{Se}$ & 0.01 & \pm 0.003 & 0.01 & 4 & $\mathrm{Rb}$ & 0.002 & \pm 0.0003 & 0.002 & 2 & & & & & \\
\hline $\mathrm{Mn}$ & 0.003 & \pm 0.001 & 0.01 & 8 & $\mathrm{Br}$ & 0.002 & \pm 0.0004 & 0.002 & 7 & & & & & \\
\hline $\mathrm{Br}$ & 0.003 & \pm 0.002 & 0.01 & 6 & & & & & & & & & & \\
\hline $\mathrm{Rb}$ & 0.002 & \pm 0.0003 & 0.002 & 2 & & & & & & & & & & \\
\hline $\mathrm{Ni}$ & 0.001 & \pm 0.0003 & 0.002 & 4 & & & & & & & & & & \\
\hline
\end{tabular}

Prom $=$ promedio aritmético, de $=$ desviación estándar, máx $=$ valor máximo; $\mathrm{N}=$ Número de datos, JA $=$ Jauja, CON $=$ Concepción, $\mathrm{HYO}=$ Huancayo. 

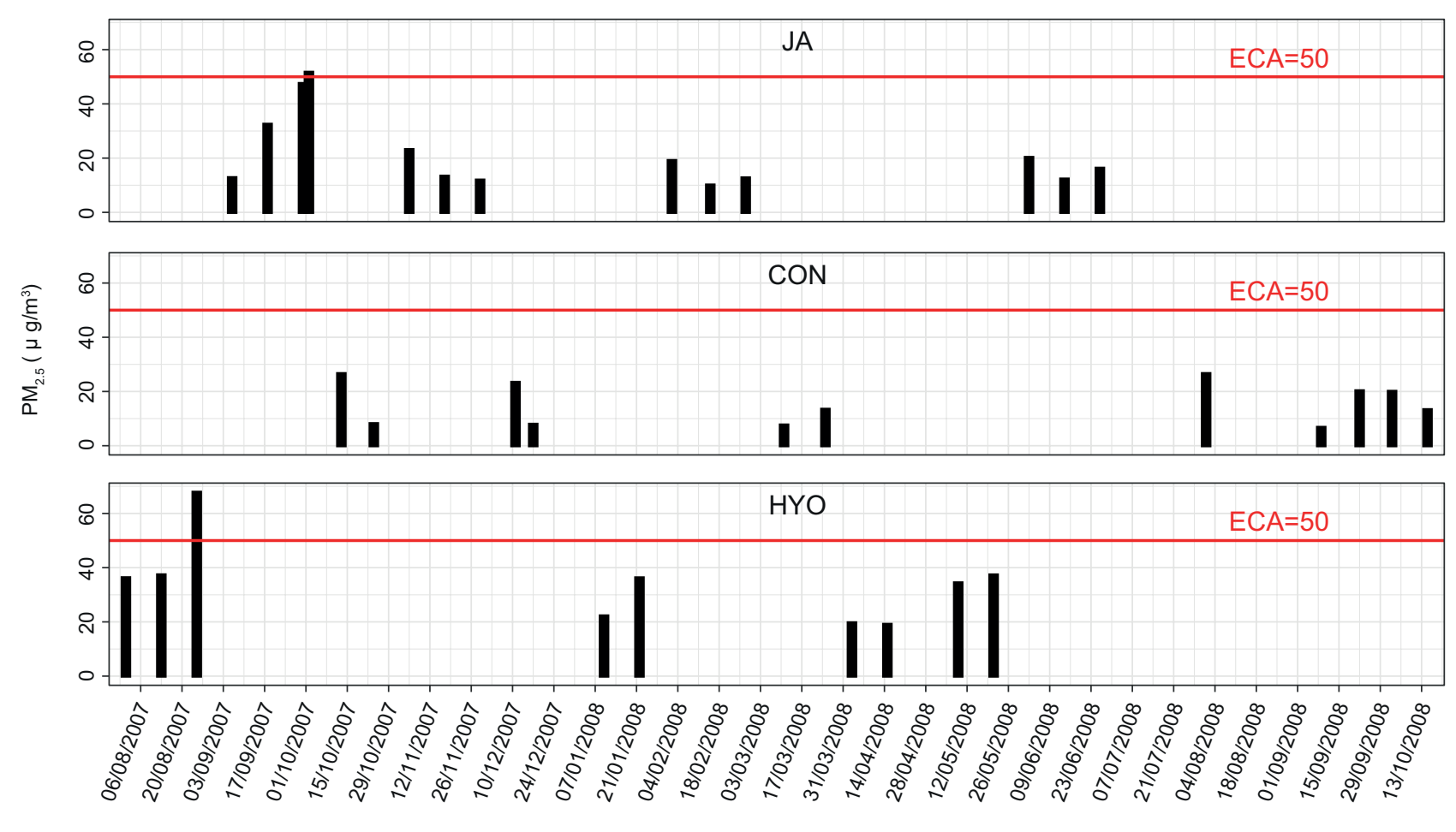

Fecha de muestreo

Fig. 5. Variación diaria de las partículas atmosféricas $\left(\mathrm{PM}_{2.5}\right)$ registradas en los centros urbanos del Valle del Mantaro $(\mathrm{JA}=\mathrm{Jauja}$, $\mathrm{CON}=$ Concepción y $\mathrm{HYO}=$ Huancayo), Perú. En la línea horizontal roja se muestra el Estándar de Calidad Ambiental peruano de $24 \mathrm{~h}\left(\mathrm{ECA}=50 \mu \mathrm{g} / \mathrm{m}^{3}\right)$.

aumenta la concentración de $\mathrm{PM}_{10}$ y $\mathrm{PM}_{2.5}$ (Suárez et al. 2017 y Huamán de la Cruz et al. 2019) y el reporte de quemas de vegetación que afectan al VM (Moya-Álvarez et al. 2017).

\section{Factor de enriquecimiento (FE) $P M_{10}$}

En la figura 6 se observan los factores de enriquecimiento determinados para los elementos químicos de $\mathrm{PM}_{10}$ en las estaciones evaluadas. Para JA, el $\mathrm{Si}, \mathrm{Al}$, Ti y Fe mostraron valores de FE menores que 1 , lo que sugiere estos elementos provienen de fuentes naturales; en cambio, los valores de FE del $\mathrm{As}, \mathrm{Pb}, \mathrm{S}, \mathrm{Zn}, \mathrm{Cu}$ y $\mathrm{Cl}$ fueron mayores que 10 , indicándonos su origen antrópico. Para el caso de $\mathrm{CON}$, el Si, Al, Fe y Ti estarían proviniendo de fuentes naturales y el $\mathrm{As}, \mathrm{Pb}, \mathrm{S}, \mathrm{Cl}, \mathrm{Zn}$ y $\mathrm{Cu}$ de fuentes antrópicas. Para la estación de HYO, sólo el Mg y el Si estarían proviniendo de fuentes naturales y el $\mathrm{As}, \mathrm{Br}, \mathrm{Pb}, \mathrm{S}, \mathrm{Zn}, \mathrm{Cl}$, y $\mathrm{Cu}$ de fuentes antrópicas. Según el FE se observa que mayor número de elementos químicos provienen de fuentes antrópicas, siendo el $\mathrm{As}, \mathrm{Pb}, \mathrm{Br}, \mathrm{S}, \mathrm{Cl}, \mathrm{Cu}$ y $\mathrm{Zn}$ los elementos de mayor factor.
PM2.5

En la figura 7 se observan los FE determinados para los elementos químicos registrados en las $\mathrm{PM}_{2.5}$. Para JA, el Si, Al, Ti, Fe, Ca y K registraron valores de $\mathrm{FE}<1$, lo que indica que provienen de fuentes naturales; en cambio, el As, $\mathrm{Pb}, \mathrm{S}$ y $\mathrm{Zn}$, mostraron FE $>10$, siendo su procedencia antrópica. Para el caso de $\mathrm{CON}$, el $\mathrm{Si}, \mathrm{Al}, \mathrm{Fe}, \mathrm{Ti}, \mathrm{Ca}$ y $\mathrm{K}$ tendrían procedencia natural y el $\mathrm{As}, \mathrm{Br}, \mathrm{S}$ y $\mathrm{Zn}$ de fuentes antrópicas. Para HYO, el Al y Fe estarían proviniendo de fuentes naturales y el $\mathrm{As}, \mathrm{Br}, \mathrm{S}$ y $\mathrm{Pb}$ de fuentes antrópicas.

Por lo tanto, al igual que en las $\mathrm{PM}_{10}$, el As, $\mathrm{Pb}$, $\mathrm{Br}, \mathrm{Zn}$ y S tendrían procedencia antrópica y el $\mathrm{Si}, \mathrm{Al}$, $\mathrm{Ti}$ y $\mathrm{Fe}$ procedencia natural.

\section{Identificación cuantitativa de las fuentes de emi- sión \\ $P M_{10}$}

En el cuadro $\mathbf{V}$ se presentan las cargas factoriales, autovalores y porcentaje de varianza del ACP para las $\mathrm{PM}_{10}$ de las estaciones de JA, CON y HYO, respectivamente.

Para JA, se identificaron cuatro componentes principales que explican el $93 \%$ de la varianza, el 

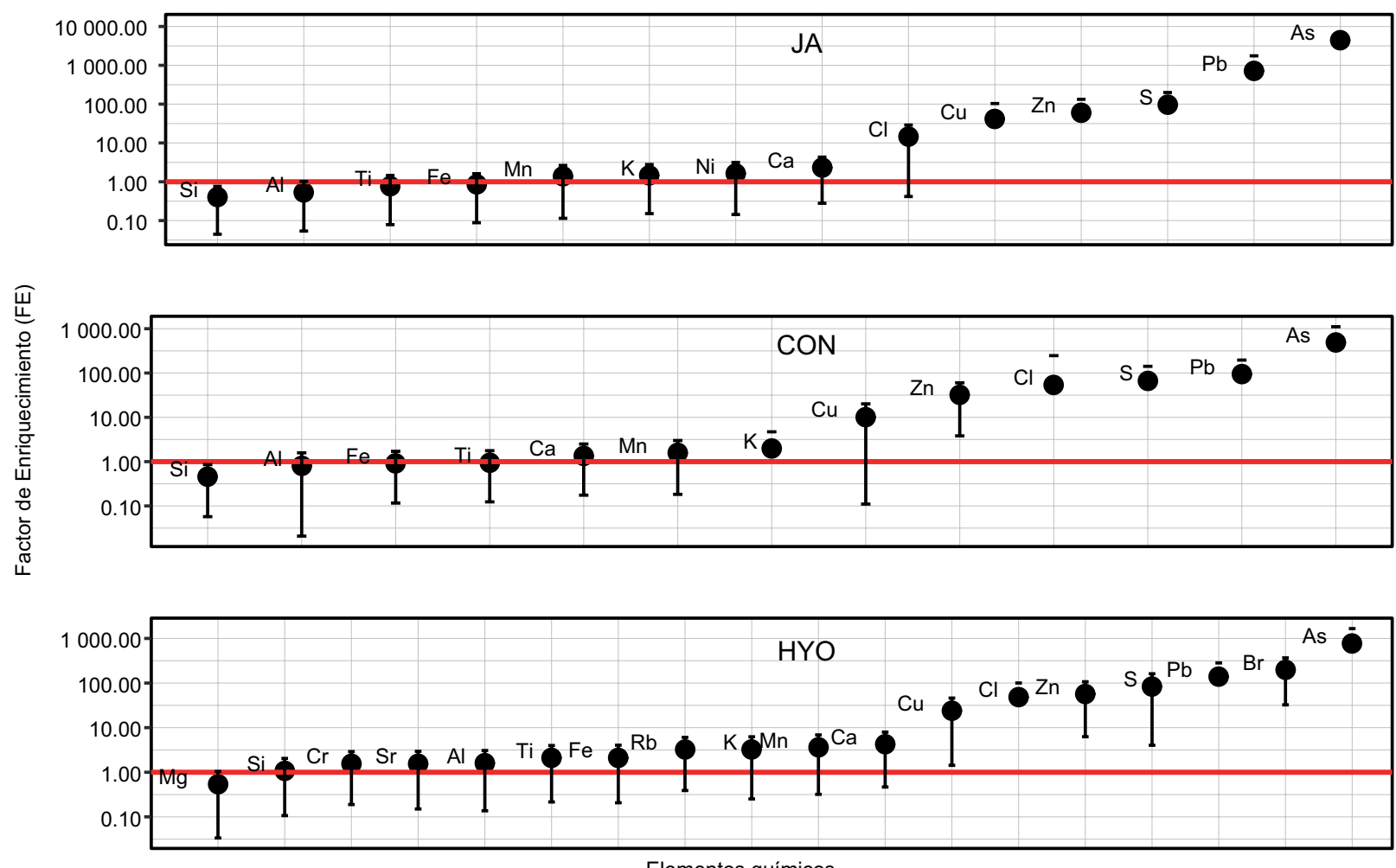

Elementos químicos

Fig. 6. Factor de enriquecimiento para los elementos químicos contenidos en las partículas atmosféricas $\left(\mathrm{PM}_{10}\right)$ registradas en los centros urbanos del Valle del Mantaro (JA = Jauja, CON = Concepción y HYO = Huancayo), Perú.
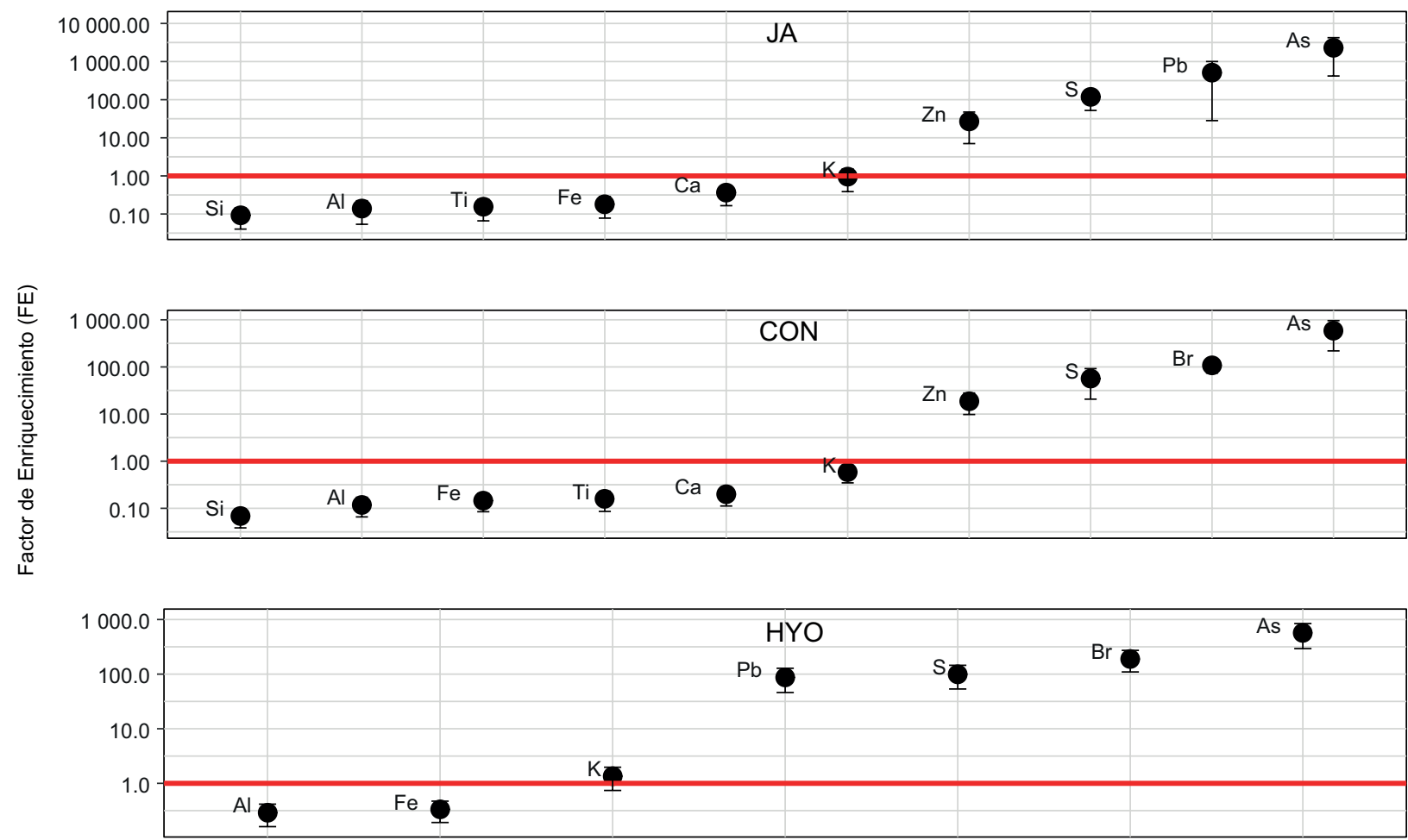

Elementos químicos

Fig. 7. Factor de enriquecimiento de los elementos químicos contenidos en las partículas atmosféricas $\left(\mathrm{PM}_{2.5}\right)$ registradas en los centros urbanos del Valle del Mantaro (JA = Jauja, CON = Concepción y HYO = Huancayo), Perú. 
FUENTES DE $\mathrm{PM}_{10} \mathrm{Y}_{\mathrm{PM}} .5$ EN EL VALLE DEL MANTARO

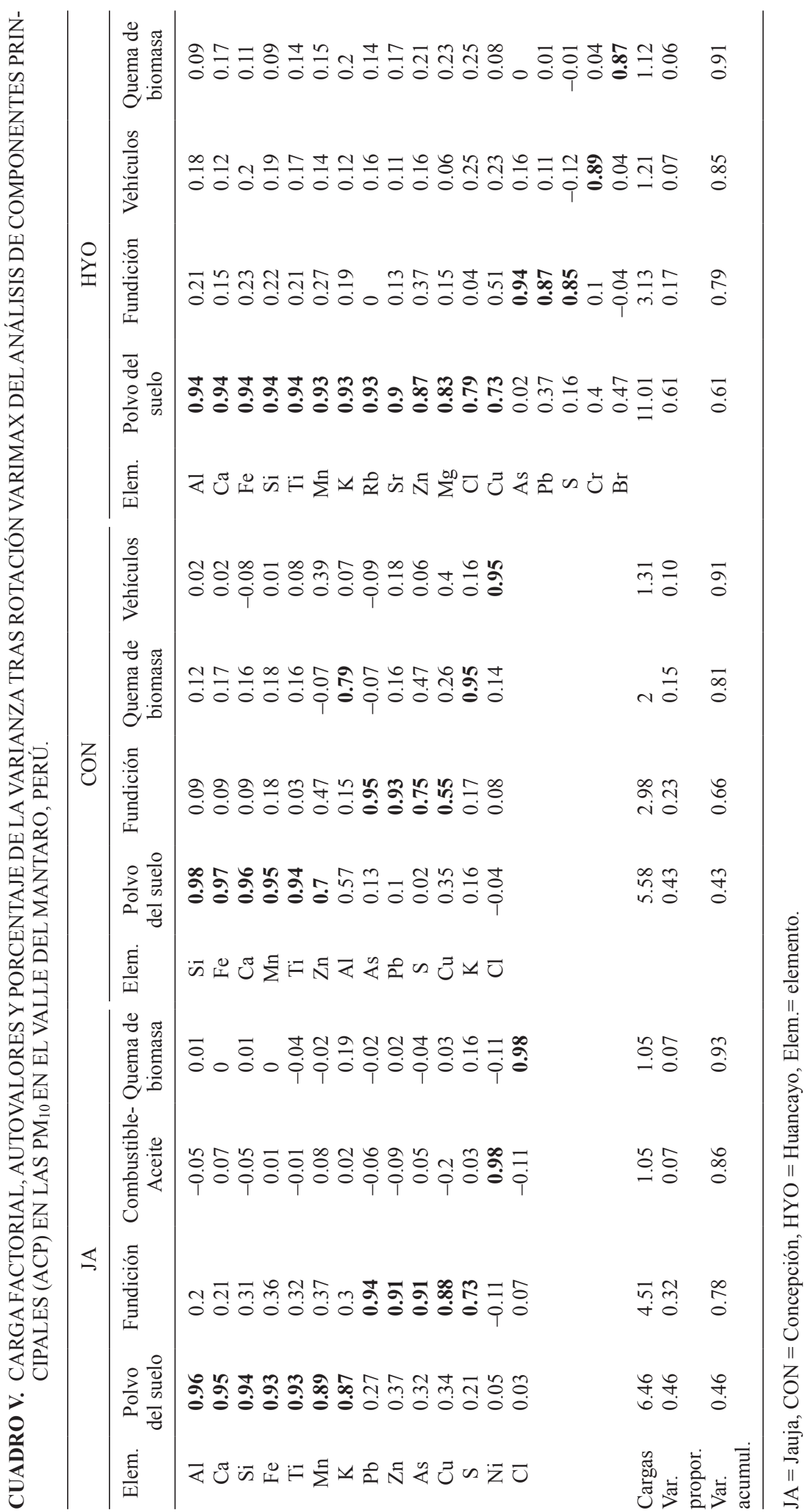


primer factor (46\% de la variabilidad) agrupa a los elementos: Al, Ca, Si, Fe, Ti, Mn y K, trazadores de polvo del suelo (Echalar et al. 1998, Artaxo et al. 1999). El segundo factor (32 \%) conformado por $\mathrm{Pb}$, $\mathrm{Zn}$, As y $\mathrm{Cu}$, fue referido a fundición (Querol et al. 2001, Querol et al. 2002 y Goix et al. 2011), dado que en la provincia de Jauja no existen industrias ni actividades relacionadas a fundiciones, la fuente más probable se encontraría ubicada a $50 \mathrm{~km}$ al noroeste del Valle del Mantaro, en la ciudad de La Oroya donde se ubica la fundición del Complejo Metalúrgico de La Oroya (CMLO), como se muestra en la figura 8. Ahí se fundían y refinaban minerales polimetálicos provenientes de los centros mineros de la zona central del Perú hasta su paralización en el año 2009. Según un estudio de Klepel (2005) existen indicios de que los contaminantes vertidos a la atmosfera por la chimenea del CMLO, estarían llegando al VM. El tercer factor $(7 \%)$, conformado por el $\mathrm{Ni}$, es atribuible al combustible-aceite (Viana et al. 2008) y el cuarto factor $(7 \%)$, conformado por el $\mathrm{Cl}$, estaría proviniendo de la quema de biomasa (Santanna et al. 2016). Como se mencionó anteriormente, en la periferia de la ciudad de Jauja se desarrollan actividades agrícolas y pecuarias donde la quema de pastizales y rastrojos es una práctica común.

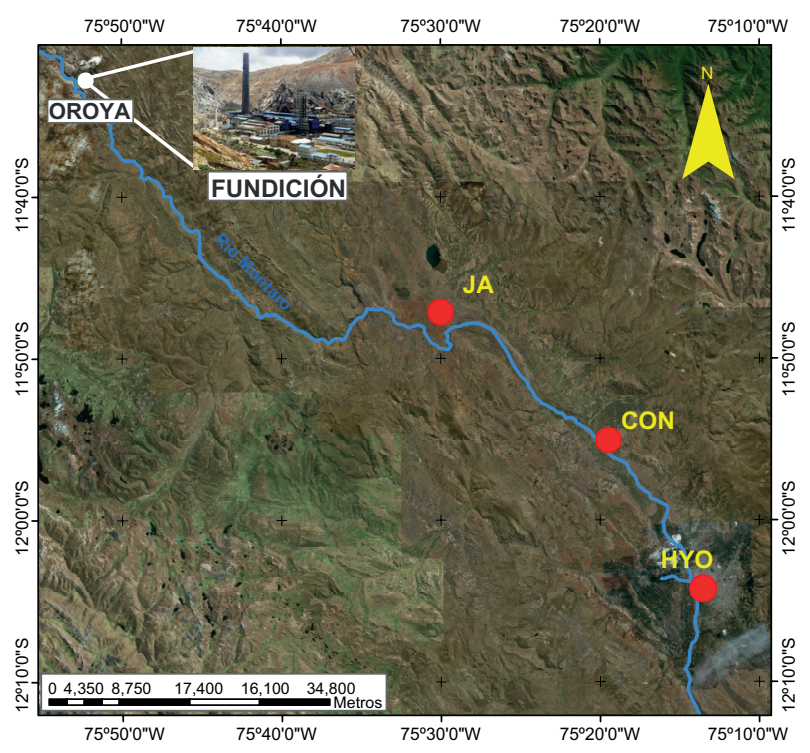

Fig. 8. Ubicación de la fundición del Complejo Metalúrgico de La Oroya y los centros urbanos del Valle del Mantaro, Perú.

Para CON, se obtuvieron cuatro componentes principales que explican el $91 \%$ de la variación, el primer factor (43\%) conformado por el $\mathrm{Si}, \mathrm{Fe}, \mathrm{Ca}$,
Al, Mn, Ti y Zn se atribuye al polvo del suelo. El segundo factor (23\%) conformado por $\mathrm{As}, \mathrm{Pb}, \mathrm{S} \mathrm{y}$ $\mathrm{Cu}$ se atribuye a la fundición del CMLO, debido a que también en la provincia de Concepción no existen industrias ni actividades relacionadas a fundiciones. El tercer factor (15\%) conformado por el Al y el $\mathrm{K}$ se atribuye a la quema de biomasa (Echalar et al. 1998). En la ciudad de Concepción una práctica común de los pobladores es el uso de leña como combustible para cocinar sus alimentos, así como la quema de pastizales y rastrojos en los trabajos de campo. El cuarto factor (10\%) conformado por el $\mathrm{Cl}$, también se agrupa con el $\mathrm{Cu}$ y el $\mathrm{Zn}$ (cargas factoriales $\approx 0.4$ ) por la significativa correlación $(\mathrm{p}<0.05)$, por lo que se atribuye a fuentes vehiculares (Stechmaun y Daunecker 1990, Viana et al. 2008), que en la ciudad están conformadas especialmente por motos y vehículos menores, que realizan servicio de transporte público en forma frecuente.

Para el caso de HYO, se obtuvieron cuatro factores principales que explican el $91 \%$ de la variabilidad. El primer factor (61\%) agrupa a los elementos $\mathrm{Al}, \mathrm{Ca}$, $\mathrm{Fe}, \mathrm{Si}, \mathrm{Ti}, \mathrm{Mn}, \mathrm{K}, \mathrm{Rb}, \mathrm{Sr}, \mathrm{Zn}, \mathrm{Mg}, \mathrm{Cl}$ y $\mathrm{Cu}$ atribuibles al polvo del suelo, el cual estaría relacionado al parque automotor, por la presencia de $\mathrm{Cl}, \mathrm{Cu}$ y $\mathrm{Zn}$ (trazadores antrópicos). El segundo factor (17\%) está conformado por el $\mathrm{As}, \mathrm{Pb}$ y S atribuible a emisiones de fundición. En Huancayo existen pequeñas fundiciones de hierro-acero y joyerías. El tercer factor (7\%), se debe al Cr atribuible a vehículos (Srimuruganandam and Shiva-Nagendra 2012a, 2012b, Banerjee et al. 2015), el cual es esperado dado que el sitio de monitoreo se ubicó en una zona de intenso tráfico vehicular. El cuarto factor (6\%) conformado por el Br es atribuible a la quema de biomasa (Echalar et al. 1998, Santanna et al. 2016). Alrededor de la ciudad de Huancayo también se desarrollan actividades agrícolas donde se queman pastizales y rastrojos agrícolas.

En la figura 9 se muestran los porcentajes de emisión para cada fuente de $\mathrm{PM}_{10}$, determinada mediante la aplicación del ACPA. En todas las estaciones el polvo del suelo fue el principal emisor de $\mathrm{PM}_{10}$ con $66 \%, 57 \%$ y $48 \%$ para JA, CON y HYO, respectivamente. Para JA (14 \%) y HYO (23\%) la quema de biomasa fue el segundo en importancia de emisión. En Concepción (7 \%) y Huancayo (10 \%) se registraron aportes de fuentes vehiculares, ello debido a que poseen mayor tráfico. Se determinaron indicios de aportes del CMLO en las $\mathrm{PM}_{10}$ registradas en Jauja (4\%) y Concepción (5\%). En cambio, para Huancayo se registraron aportes de fundiciones locales en un $13 \%$. Llama la atención que en la ciudad de Concepción el porcentaje no identificado de fuentes (Otros) 

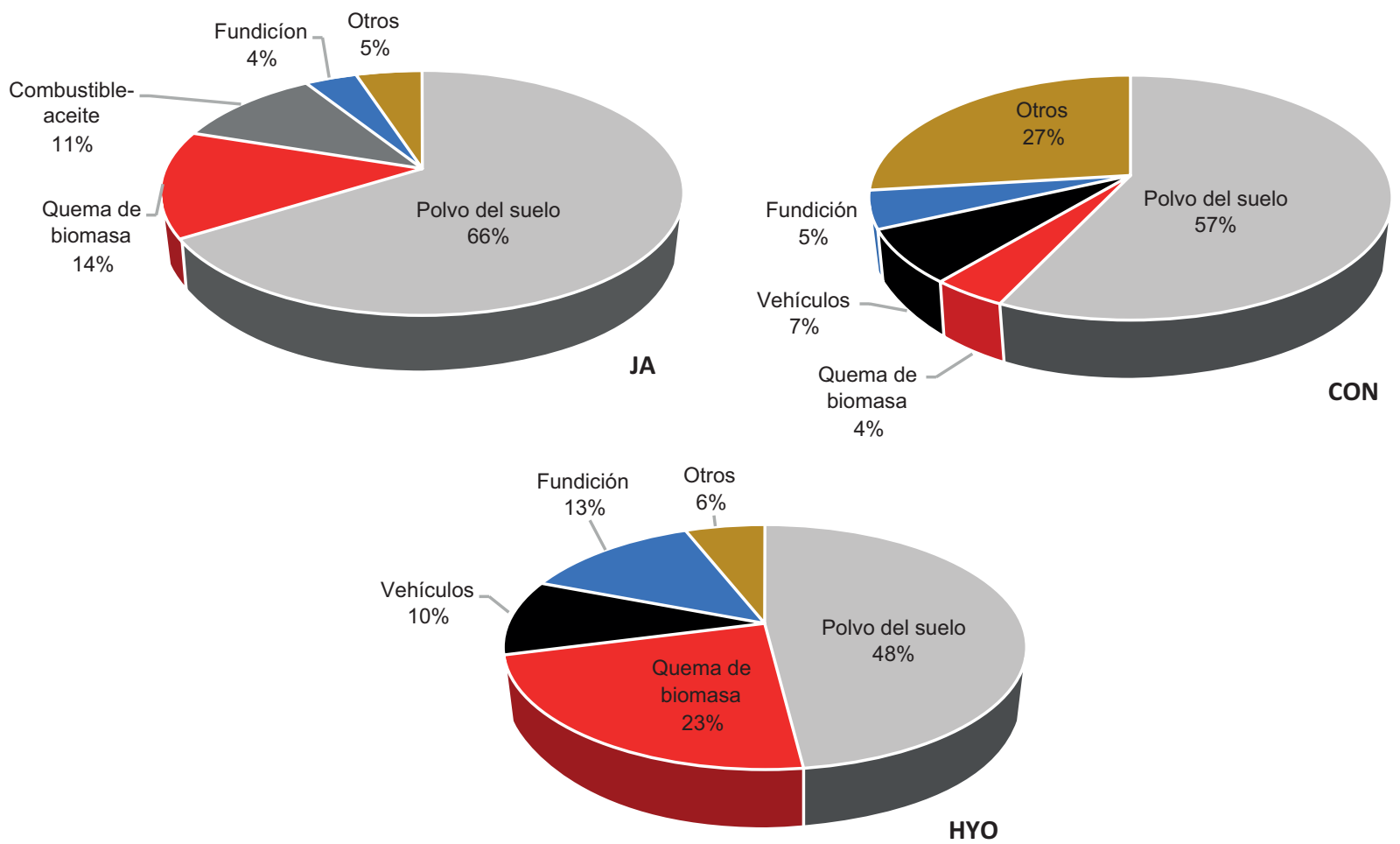

Fig. 9. Porcentajes de emisión por fuentes de partículas atmosféricas $\left(\mathrm{PM}_{10}\right)$ estimadas por el análisis de componentes principales absoluto para los centros urbanos del Valle del Mantaro (JA = Jauja, CON = Concepción y HYO = Huancayo), Perú.

ocupe el $27 \%$ de aporte, ello quizás se deba a que es una zona de convergencia de vientos para el valle del Mantaro (Flores et al. 2019).

Las fuentes determinadas fueron confirmadas por el agrupamiento de los elementos químicos en los dendrogramas que se muestran en la figura 10. Para el caso de JA se determinaron cuatro grupos: G1, con elementos trazadores de polvo del suelo
(K, Al, Si, Fe, Ti, Ca y Mn), G2, con elementos trazadores de fundición ( $\mathrm{S}, \mathrm{As}, \mathrm{Cu}, \mathrm{Pb}$ y $\mathrm{Zn}$ ), G3 con un elemento trazador de quema de biomasa $(\mathrm{Cl})$ y G4, con un elemento trazador de combustible-aceite (Ni). En CON, se determinaron cinco grupos: G1, con elementos trazadores de fundición ( $\mathrm{S}$, As y $\mathrm{Pb}$ ), $\mathrm{G} 2$, con un elemento trazador de quema de biomasa (Cl), G3, con elementos trazadores de vehículos $(\mathrm{Cu}$
$J A$

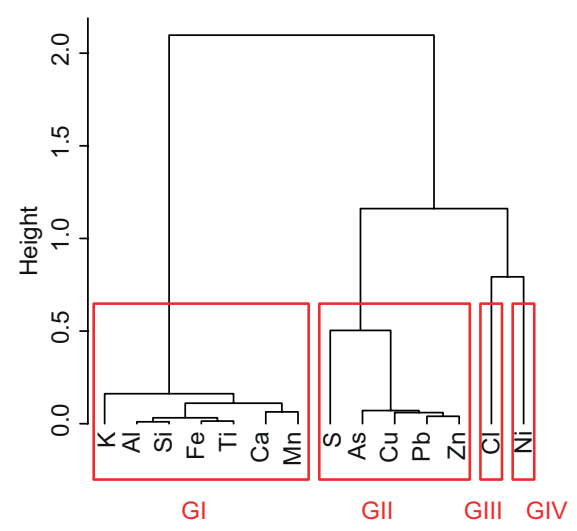

CON

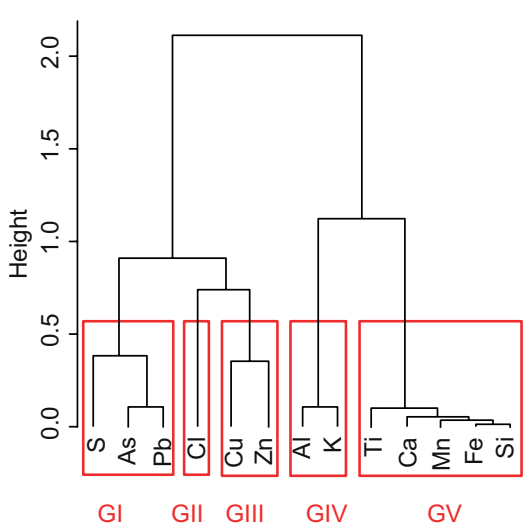

HYO

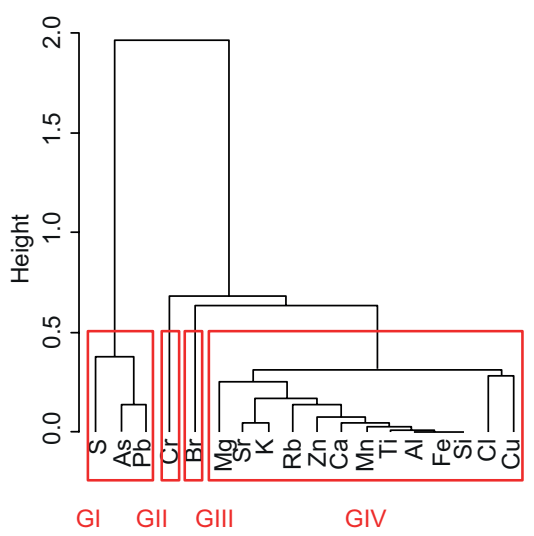

Fig. 10. Dendrograma para los elementos químicos contenidos en las partículas atmosféricas $\left(\mathrm{PM}_{10}\right)$ registradas en los centros urbanos del Valle del Mantaro (JA = Jauja, CON = Concepción y HYO = Huancayo), Perú. 
CUADRO VI. CARGA FACTORIAL, AUTOVALORES Y PORCENTAJE DE LA VARIANZA TRAS ROTACIÓN VARIMAX DEL ANÁLISIS DE COMPONENTES PRINCIPALES (ACP) EN LAS PM 2.5 EN EL VALLE DEL MANTARO, PERÚ.

\begin{tabular}{|c|c|c|c|c|c|c|c|c|c|c|}
\hline \multicolumn{3}{|c|}{$\mathrm{JA}$} & \multicolumn{3}{|c|}{$\mathrm{CON}$} & \multicolumn{5}{|c|}{ HYO } \\
\hline Elemento & $\begin{array}{c}\text { Polvo } \\
\text { del suelo }\end{array}$ & Fundición & Elemento & $\begin{array}{c}\text { Polvo } \\
\text { del suelo }\end{array}$ & Fundición & Elemento & $\begin{array}{c}\text { Polvo } \\
\text { del suelo }\end{array}$ & Fundición & $\begin{array}{c}\text { Quema } \\
\text { de biomasa }\end{array}$ & Vehículos \\
\hline $\mathrm{Al}$ & 0.99 & -0.14 & $\mathrm{Al}$ & 0.95 & 0.27 & $\mathrm{Fe}$ & 0.87 & 0.39 & 0.26 & -0.08 \\
\hline $\mathrm{Ca}$ & 0.99 & 0.03 & $\mathrm{Si}$ & 0.93 & 0.35 & $\mathrm{Al}$ & 0.81 & 0.36 & 0.45 & -0.08 \\
\hline $\mathrm{Fe}$ & 0.99 & 0.06 & $\mathrm{Fe}$ & 0.9 & 0.29 & $\mathrm{~K}$ & 0.59 & 0.34 & 0.55 & 0.47 \\
\hline $\mathrm{Si}$ & 0.99 & -0.02 & $\mathrm{Ca}$ & 0.87 & 0.48 & $\mathrm{~Pb}$ & 0.3 & 0.92 & 0.15 & 0.14 \\
\hline $\mathrm{Ti}$ & 0.99 & 0.07 & $\mathrm{~K}$ & 0.73 & 0.57 & As & 0.36 & 0.85 & 0.23 & 0.25 \\
\hline $\mathrm{K}$ & 0.62 & 0.28 & $\mathrm{~S}$ & 0.4 & 0.88 & $\mathrm{Br}$ & 0.35 & 0.19 & 0.91 & 0.08 \\
\hline As & -0.04 & 0.99 & As & 0.28 & 0.95 & $\mathrm{~S}$ & -0.08 & 0.18 & 0.06 & 0.98 \\
\hline $\mathrm{Zn}$ & 0.01 & 0.98 & & & & & & & & \\
\hline $\mathrm{Pb}$ & -0.03 & 0.98 & & & & & & & & \\
\hline $\mathrm{S}$ & 0.19 & 0.79 & & & & & & & & \\
\hline Cargas & 5.32 & 3.64 & & 4.11 & 2.52 & & 2.11 & 2.04 & 1.48 & 1.27 \\
\hline Var. proporción & 0.53 & 0.36 & & 0.59 & 0.36 & & 0.3 & 0.29 & 0.21 & 0.18 \\
\hline Var. acumulada & 0.53 & 0.90 & & 0.59 & 0.95 & & 0.3 & 0.59 & 0.80 & 0.99 \\
\hline
\end{tabular}

$\mathrm{JA}=$ Jauja, $\mathrm{CON}=$ Concepción, $\mathrm{HYO}=$ Huancayo.

y Zn), y G4 y GV con elementos trazadores de suelo (Al y K, y Ti, Ca, Mn, Fe y Si, respectivamente). En HYO se determinaron cuatro grupos: G1, con elementos trazadores de fundición ( $\mathrm{S}$, As y $\mathrm{Pb}$ ), G2, con un elemento trazador de vehículos $(\mathrm{Cr}), \mathrm{G} 3$, con un elemento trazador de quema de biomasa (Br), y el G4 con elementos trazadores de suelo y vehículos ( $\mathrm{Mg}, \mathrm{Sr}, \mathrm{K}, \mathrm{Rb}, \mathrm{Zn}, \mathrm{Ca}, \mathrm{Mn}, \mathrm{Ti}, \mathrm{Al}, \mathrm{Fe}, \mathrm{Si}, \mathrm{Cl}$ y Cu ). Lo anterior confirma que existe un mayor número de fuentes antrópicas de $\mathrm{PM}_{10}$ en cada lugar estudiado.

\section{PM2.5}

En el cuadro VI se presentan las cargas factoriales, autovalores y porcentaje de varianza del ACP para $\mathrm{PM}_{2.5}$ de las estaciones de JA, CON y HYO. Para Jauja, se identificaron dos componentes principales que explican el $90 \%$ de la varianza, el primer factor (53\% de la variabilidad) agrupa a los elementos $\mathrm{Al}$, $\mathrm{Ca}, \mathrm{Fe}, \mathrm{Si}$, Ti y K, trazadores de polvo del suelo. El segundo factor (36\%) conformado por As, Zn, $\mathrm{Pb}$ y $\mathrm{S}$, atribuibles a la fundición del CMLO. Para Concepción, también se obtuvieron dos componentes principales que explican el $95 \%$ de la variación, el primer factor ( $59 \%$ de la variabilidad) conformado por el $\mathrm{Al}, \mathrm{Si}, \mathrm{Fe}, \mathrm{Ca}$ y $\mathrm{K}$ es atribuible al polvo del suelo; el segundo factor (36\%) conformado por S y As fue atribuido a la fundición del CMLO. Para el caso de Huancayo, se obtuvieron cuatro factores principales que explican el $99 \%$ de la variabilidad. El primer factor (30\% de la variabilidad) agrupa a los elementos $\mathrm{Fe}$, Al y K atribuibles al polvo del suelo.
El segundo factor (29\%) está conformado por el As y $\mathrm{Pb}$ atribuibles a fundición. El tercer factor $(21 \%)$, donde tenemos al $\mathrm{Br}$ es atribuible a la quema de biomasa y el cuarto factor (18\%) conformado por el $\mathrm{S}$ atribuible a vehículos.

En la figura 11 se muestran los porcentajes de emisión para cada fuente de $\mathrm{PM}_{2.5}$, determinada mediante la aplicación del ACPA. En todas las estaciones el polvo del suelo fue el mayor emisor con $50 \%, 73 \%$ y $34 \%$ para JA, CON y HYO, respectivamente. Para JA (33 \%) y CON (23\%) la fundición fue el segundo en importancia de emisión, seguidos de fuentes indeterminadas. Para HYO la quema de biomasa fue la segunda en importancia de aporte $(27 \%)$ seguida de vehículos $(26 \%)$; el aporte de las fundiciones locales fue del $7 \%$.

Los dendrogramas obtenidos con los elementos químicos de las $\mathrm{PM}_{2.5}$ se muestran en la figura 12. Para el caso de JA se determinaron dos grupos: G1, con elementos trazadores de fundición $(\mathrm{S}, \mathrm{Pb}, \mathrm{As}$, y Zn) y G2, con elementos trazadores de polvo del suelo (K, Al, Ca, Ti, Fe y Si). En CON, también se determinaron dos grupos: G1 con elementos trazadores de polvo del suelo ( $\mathrm{Br}, \mathrm{K}, \mathrm{Fe}, \mathrm{Ca}, \mathrm{Al}$ y $\mathrm{Si}$ ) y $\mathrm{G} 2$, con elementos trazadores de fundición (As, $\mathrm{S}$, $\mathrm{Zn}$ y Ti). En HYO se determinaron cuatro grupos: G1 con un elemento trazador de vehículos (S), G2, con elementos trazadores de fundición (As y $\mathrm{Pb}$ ), G3, con elementos trazadores de polvo del suelo (A1 y Fe), y G4, con elementos trazadores de quema de biomasa (Br y K). 

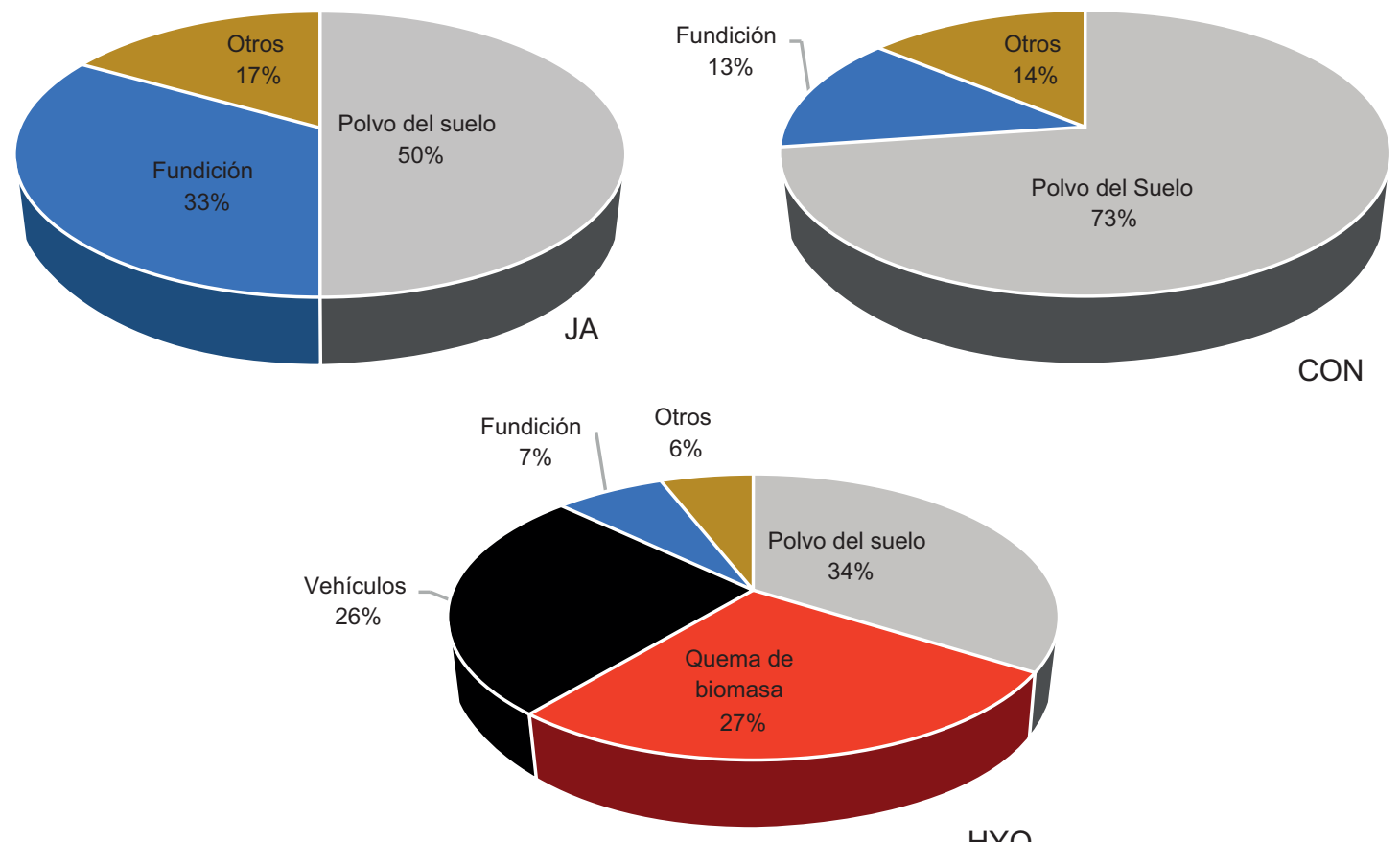

HYO

Fig. 11. Porcentajes de emisión por fuentes de partículas atmosféricas $\left(\mathrm{PM}_{2.5}\right)$ estimadas por el análisis de componentes principales absoluto para los centros urbanos del Valle del Mantaro $(\mathrm{JA}=\mathrm{Jauja}, \mathrm{CON}=\mathrm{Concepción}$ y $\mathrm{HYO}=$ Huancayo), Perú.

JA

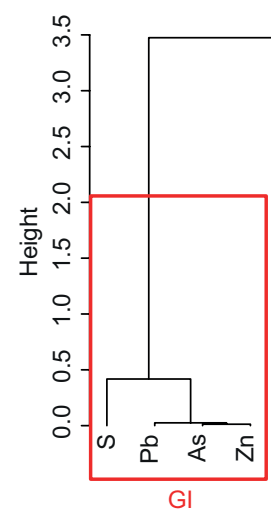

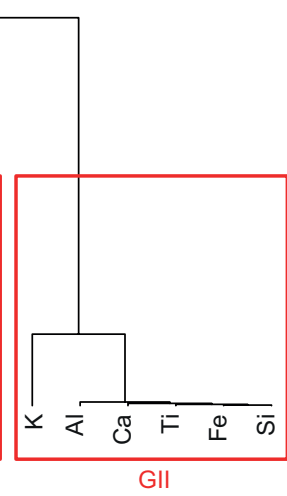

CON

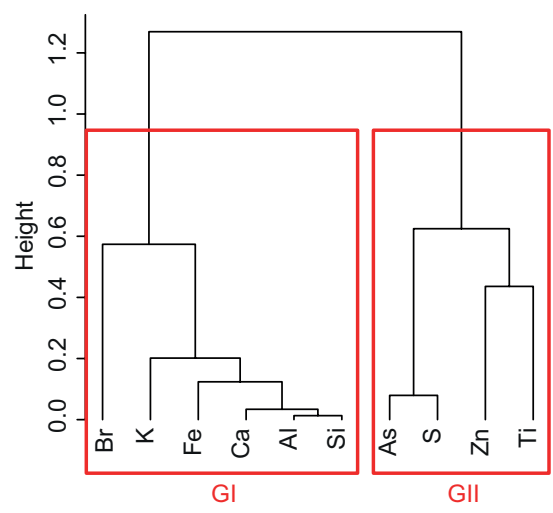

HYO

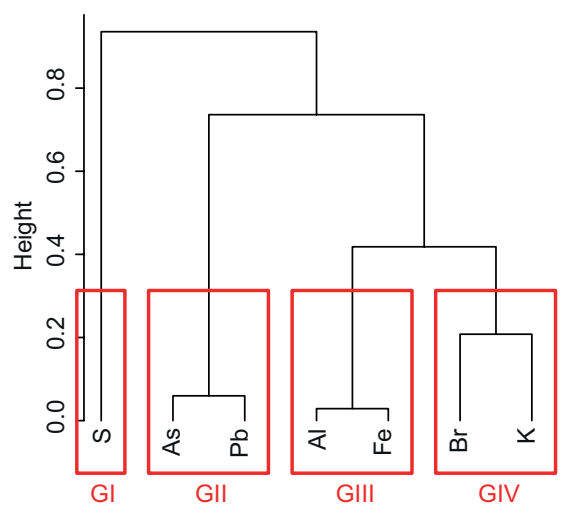

Fig. 12. Dendrogramas para los elementos químicos contenidos en las partículas atmosféricas $\left(\mathrm{PM}_{2.5}\right)$ de los centros urbanos del Valle del Mantaro (JA = Jauja, CON = Concepción y HYO = Huancayo $)$, Perú.

Estos resultados demuestran que a nivel de las $\mathrm{PM}_{10}$ y $\mathrm{PM}_{2.5}$ de los centros urbanos del VM se tienen aportes de fuentes antrópicas y naturales, siendo mayor el número de fuentes antrópicas, cuya contribución se puede controlar o disminuir a través de la implementación de planes y programas de gestión de calidad de aire.

\section{CONCLUSIONES}

Se determinó que las concentraciones $\mathrm{PM}_{10} \mathrm{y}$ $\mathrm{PM}_{2.5}$ superan a las de la normatividad peruana vigente, lo que demuestra que las partículas atmosféricas representa un riesgo para los pobladores de los centros urbanos del VM. Siendo la ciudad de Huancayo 
la que registró mayor problemática por superar más veces los límites establecidos.

A través de la aplicación de FE se obtuvieron elementos trazadores de fuentes naturales $(\mathrm{FE}<1)$ : $\mathrm{Si}, \mathrm{Al}$, Ti y Fe y elementos de origen antrópico (FE $>$ 10): $\mathrm{As}, \mathrm{Pb}, \mathrm{Br}, \mathrm{Zn}$ y $\mathrm{S}$, tanto en $\mathrm{PM}_{10}$ como en $\mathrm{PM}_{2.5}$.

En el caso de las fuentes de emisión de PM del VM en total se han registrado cinco fuentes: polvo del suelo (Al, $\mathrm{Ca}, \mathrm{Si}, \mathrm{Fe}, \mathrm{Ti}, \mathrm{Mn}$ y K ), quema de biomasa $(\mathrm{Cl}, \mathrm{Br}, \mathrm{K})$, vehículos $(\mathrm{Cu}, \mathrm{Zn}, \mathrm{Cl}, \mathrm{Cr})$, combustibleaceite $(\mathrm{Ni})$ y fundición $(\mathrm{Pb}, \mathrm{Zn}$, As y $\mathrm{Cu}$ ). Se logró determinar mayor número de fuentes de $\mathrm{PM}_{10}$ por el mayor número de datos registrados.

La principal fuente de emisión de $\mathrm{PM}_{10}$ y $\mathrm{PM}_{2.5}$ para todas las áreas urbanas evaluadas fue el polvo del suelo, seguida de las quemas de biomasa, lo que demuestra la influencia de áreas rurales circundantes y las quemas en zonas agrícolas y ganaderas. También las emisiones del parque automotor y la quema de combustible-aceite tienen gran importancia.

Otra fuente de importancia para las poblaciones del VM fue la fundición, a la que están asociados dos elementos tóxicos para la salud de la población como el $\mathrm{Pb}$ y el As.

\section{AGRADECIMIENTOS}

Al Arzobispado de Huancayo y Mesa de Diálogo Ambiental de la Región Junín (MEDIAREJ) y a su proyecto "Fortalecimiento de la gestión ambiental para la lucha contra la contaminación de la zona alta-media de la cuenca del río Mantaro-Junín", conocido como "El Mantaro Revive" el cual fue financiado por el Fondo Ítalo Peruano (FIP). A la United States Agency for International Development (USAID) de los EUA vía el proyecto "Impacto del transporte transfronterizo de contaminantes del aire sobre los Andes Centrales de Perú relacionados a la quema de vegetación en la Amazonía" del programa PEER Sciences. Apreciamos el aporte de los revisores que mejoraron el manuscrito.

\section{REFERENCIAS}

Andreae M.O. y Crutzen P.J. (1997). Atmospheric aerosols: biogeochemical sources and role in atmospheric chemistry. Science 276, 1052-1058. https:// doi.org/10.1126/science.276.5315.1052

Artaxo P., Oyola P. y Martinez, R. (1999). Aerosol composition and source apportionment in Santiago de Chile. Nucl. Instruments Methods Phys. Res. 150, 409-416. https://doi.org/10.1016/S0168-583X(98)01078-7
Banerjee T., Murari V., Kumar M. y Raju, M.P. (2015). Source apportionment of airborne particulates through receptor modeling: Indian scenario. Atmos. Res. 164, 167-187. https://doi.org/10.1016/j.atmosres.2015.04.017

Belis C.A., Larsen B.R., Amato F., Haddad E., Favez O., Harrison R.M., Hopke P.K., Nava S., Paatero P., Prévôt A., Quass U. y Vecchi R. (2014). European guide on air pollution source apportionment with receptor models. European Union, Luxemburgo, 88 pp. https://doi. org/10.2788/9307

Caiazzo F., Ashok A., Waitz I.A., Yim S.H.L. y Barrett S.R.H. (2013). Air pollution and early deaths in the United States . Part I : Quantifying the impact of major sectors in 2005. Atmos. Environ. 79, 198-208. https:// doi.org/10.1016/j.atmosenv.2013.05.081

Carbajal-Arroyo L., Barraza-Villarreal A., Durand-Pardo R., Moreno-Macías H., Espinoza-Laín R., ChiarellaOrtigosa P. y Romieu I., (2007). Impact of traffic flow on the asthma prevalence among school children in Lima, Peru. J. Asthma 44, 197-202. https://doi. org/10.1080/02770900701209756

Chavent M., Guegan H., Kuentz V., Patouille B. y Saracco J. (2009). PCA and PMF based methodology for air pollution sources identification and apportionment. Environmetrics 20 (8), 928-942. https://doi.org/10.1002/env.963

Chester R., Nimmo M., Fones G.R., Keyse S. y Zhang Z. (2000). Trace metal chemistry of particulate aerosols from the UK mainland coastal rim of the NE Irish sea. Atmos. Environ. 34 (6), 949-958. https://doi. org/10.1016/S1352-2310(99)00234-4

Cohen A.J., Brauer M., Burnett R., Anderson H.R., Frostad J., Estep K., Balakrishnan K., Brunekreef B., Dandona L., Dandona R., Feigin V., Freedman G., Hubbell B., Jobling A., Kan H., Knibbs L., Liu Y., Martin R., Morawska L., Pope C.A., Shin H., Straif K., Shaddick G., Thomas M., van Dingenen R., van Donkelaar A., Vos T., Murray C.J.L. y Forouzanfar M.H. (2017). Estimates and 25-year trends of the global burden of disease attributable to ambient air pollution: an analysis of data from the Global Burden of Diseases Study 2015. The Lancet 389, 1907-1918. https://doi. org/10.1016/S0140-6736(17)30505-6

CONAM (2005). Plan de acción para la prevención y descontaminación del aire en la cuenca atmosférica de Huancayo, 2006 - 2010. Concejo Nacional del Ambiente. Plan. Huancayo,Perú, 48 pp.

Davidson C.I., Phalen R.F. y Solomon P.A. (2005). Airborne particulate matter and human Health: A review. Aerosol Sci. Technol. 39, 737-749. https://doi. org/10.1080/02786820500191348

Echalar F., Artaxo P., Vanderlei Martins J., Yamasoe M. y Gerab F. (1998). Long-term monitoring of atmospheric 
aerosols in the Amazon Basin: Source identification and apportionment. J. Geophys. Res. 103 (D24),3184931864. https://doi.org/10.1029/98JD01749

Estevan R., Martínez-Castro D., Suarez-Salas L., Moya A. y Silva Y. (2019). First two and a half years of aerosol measurements with an AERONET sunphotometer at the Huancayo Observatory, Peru. Atmos. Environ. X 3, 1-13.:https://doi.org/10.1016/j. aeaoa.2019.100037

Flores-Rojas J.L., Moya-Alvarez A.S., Kumar S., Martinez-Castro D., Villalobos-Puma E. y Silva-Vidal Y. (2019). Analysis of possible triggering mechanisms of severe thunderstorms in the tropical central Andes of Peru, Mantaro Valley. Atmosphere 10 (6), 1-29. https:// doi.org/10.3390/atmos10060301

Gidhagen L., Kahelin H., Schmidt-Thomé P. y Johansson C. (2002). Anthropogenic and natural levels of arsenic in PM10 in central and northern Chile. Atmos. Environ. 36 (23), 3803-3817. https://doi.org/10.1016/ S1352-2310(02)00284-4

Gilardoni S., Vignati E., Marmer E., Cavalli F., Belis C., Gianelle V; Loureiro A. y Artaxo P. (2011). Sources of carbonaceous aerosol in the Amazon Basin. Atmos. Chem. Phys. 11 (6), 2747-2764. https://doi. org/10.5194/acp-11-2747-2011

Goix S., Point D., Oliva P., Polve M., Duprey J.L., Mazurek H., Guislain L., Huayta C., Barbieri F.L. y Gardon J. (2011). Influence of source distribution and geochemical composition of aerosols on children exposure in the large polymetallic mining region of the Bolivian Altiplano. Sci. Total Environ. 412-413, 170184. https://doi.org/10.1016/j.scitotenv.2011.09.065

Hamanaka R.B. y Mutlu G.M. (2018). Particulate matter air pollution: effects on the cardiovascular system. Front. Endocrinol (Lausanne) 9, 1-15. https://doi. org $/ 10.3389 /$ fendo. 2018.00680

Hime N.J., Marks G.B. y Cowie C.T. (2018). A comparison of the health effects of ambient particulate matter air pollution from five emission sources. Int. J. Environ. Res. Public Health 15 (6), 1-24. https://doi. org/10.3390/ijerph15061206

Hopke P.K. (2010). The application of receptor modeling to air quality data. Pollution Atmosphérique, 91 - 109.

Huamán de la Cruz A., Bendezu Roca Y., Suarez-Salas L., Pomalaya J., Alvarez-Tolentino D. y Gioda A. (2019). Chemical characterization of $\mathrm{PM}_{2.5}$ at rural and urban sites around the Metropolitan Area of Huancayo, Peru. Atmosphere 10 (1), 1-17. https://doi.org/10.3390/ atmos 10010021

Husson F., Josse J. y Pages, J. (2010). Principal component methods-hierarchical clustering-partitional clustering: why would we need to choose for visualizing data? Tech. Report-Agrocampus, 1-17.
IGP (2005). Atlas climático de precipitación y temperatura del aire en la Cuenca del río Mantaro. Instituto Geofísico del Perú. Lima, Perú, 107 pp.

Kaiser H.F. (1958). The varimax criterion for analytic rotation in factor analysis. Psychometrika 23, 187-200. https://doi.org/10.1007/BF02289233

Klepel R. (2005). Estudio de saturación con tubos pasivos en la ciudad de La Oroya. Klepel Consulting S.A.C., Lima, Perú, 71 pp.

Meszaros E. (1999). Fundamentals of atmospheric aerosol chemistry. Akademiai Kiado, Budapest, p. 549. https:// doi.org/10.1023/A:1010753724116

MINAM (2010). Compendio de la legislación ambiental peruana. 1a ed., vol. I, Marco Normativo General. Ministerio del Ambiente. Registro. Lima, Perú, 281 pp.

MINAM (2017). Decreto Supremo 003-2017-MINAM. Aprueban Estándares de Calidad Ambiental (ECA) para aire y establecen Disposiciones Complementarias. Ministerio del Ambiente. Diario Oficial del Peruano. 7 de junio del 2017.

Moya-Alvarez A.S., Arredondo R.E. y Yuli-Posadas, R.Á. (2017). Determinación de la presencia de partículas $\left(\mathrm{PM}_{10}\right)$ en Perú producidas por quema de biomasa con ayuda de modelos numéricos. Rev. Int. Contam. Ambient. 33 (1), 99-108. https://doi.org/10.20937/ RICA.2017.33.01.09

Olivier B. y David R. (2013). Clouds and aerosols. En: Climate Change 2013. The Physical Science Basis: Working Group I. Contribution to the Fifth Assessment Report of the Intergovernmental Panel on Climate Change (Intergovernmental Panel on Climate Change, Ed.).Cambridge University, Reino Unido y Nueva York, EUA, pp. 571-658. https://doi.org/10.1017/ CBO9781107415324.016

OMS (2006). Guías de calidad de aire de la OMS relativas al material particulado, el ozono, el dióxido de nitrógeno y el dióxido de azufre: Resumen de evaluación de riesgos. Organización Mundial de la Salud. Ginebra, Suiza, 20 pp.

OMS (2018). 9 out of 10 people worldwide breathe polluted air, but more countries are taking action. Organizacion Mundial de la Salud [en línea]. http://bitly. com/whoair9 01/06/2020

Pant P. y Harrison R.M. (2013). Estimation of the contribution of road traffic emissions to particulate matter concentrations from field measurements: A review. Atmos. Environ. 77, 78-97. https://doi.org/10.1016/j. atmosenv.2013.04.028

Pison G., Rousseeuw P.J., Filzmoser P. y Croux C. (2003). Robust factor analysis. J. Multivar. Anal. 84, 145-172. https://doi.org/10.1016/S0047-259X(02)00007-6

Pöschl U. (2005). Atmospheric aerosols: Composition, transformation, climate and health effects. Angewandte 
Chemie 44 (46), 7520-7540. https://doi.org/10.1002/ anie. 200501122

Querol X., Alastuey A., Rodriguez S., Plana F., Ruiz C.R., Cots N., Massagué G. y Puig O. (2001). $\mathrm{PM}_{10}$ and $\mathrm{PM}_{2.5}$ source apportionment in the Barcelona Metropolitan area, Catalonia, Spain. Atmos. Environ. 35 (36), 6407-6419. https://doi.org/10.1016/S13522310(01)00361-2

Querol X., Alastuey A., de la Rosa J., Sánchez-de-laCampa A., Plana F. y Ruiz, C.R. (2002). Source apportionment analysis of atmospheric particulates in an industrialised urban site in southwestern Spain. Atmos. Environ. 36 (19), 3113-3125. https://doi.org/10.1016/ S1352-2310(02)00257-1

Querol X. (2008). Calidad del aire, partículas en suspensión y metales. Rev. Esp. Salud Pública 82 (5), 447-454. https://doi.org/10.1590/S1135-57272008000500001

R Core Team (2015). R : A language and environment for statistical computing. R Foundati. Vienna [en línea]. http://www.R-project.org/ 27/11/2019

Rahn K.A. (1971). Sources of trace elements in aerosols: an approach to clean air. Dept. of Meteorology and Oceanography, The University of Michigan. doi:10.2172/4030150 [en línea] https://digital.library. unt.edu/ark:/67531/metadc870731/ 02/06/2020.

Reddington C.L., Morgan W.T., Darbyshire E., Brito J., Coe H., Artaxo P., Marsham J. y Spracklen D. V. (2019). Biomass burning aerosol over the Amazon: analysis of aircraft, surface and satellite observations using a global aerosol model. Atmos. Chem. Phys. 19 (14), 9125-9152. https://doi.org/10.5194/acp-199125-2019

Santanna F.B., De Almeida Filho E.O., Vourlitis G.L., De Arruda P.H.Z., Da Silva-Palácios R. y de SouzaNogueira J. (2016). Elemental composition of $\mathrm{PM}_{10}$ and $\mathrm{PM}_{2.5}$ for a savanna (cerrado) region of Southern Amazonia. Quim. Nova 39 (10), 1170-1176. https:// doi.org/10.21577/0100-4042.20160154

Srimuruganandam B. y Shiva-Nagendra S.M. (2012a). Application of positive matrix factorization in characterization of $\mathrm{PM}_{10}$ and $\mathrm{PM}_{2.5}$ emission sources at urban roadside. Chemosphere 88 (1), 120-130. https:// doi.org/10.1016/j.chemosphere.2012.02.083
Srimuruganandam B. y Shiva Nagendra S.M. (2012b). Source characterization of $\mathrm{PM}_{10}$ and $\mathrm{PM}_{2.5}$ mass using a chemical mass balance model at urban roadside. Sci. Total Environ. 433 (1), 8-19. https://doi.org/10.1016/j. scitotenv.2012.05.082

Stechmaun H. y Daunecker W. (1990). Characterization and source analysis of vehicle-generated aerosols. J. Aerosol Sci. 21 (1), 287-290. https://doi. org/10.1016/0021-8502(90)90240-X

Suarez-Salas L., Torres C., Helmig D. y Hueber J. (2017). Medición y analisis del aerosol de carbono negro en el Observatorio de Huancayo, Perú. Rev. Boliv. Física 30 (30), 7-15.

Suárez-Salas L., Alvarez-Tolentino D., Bendezú Y. y Pomalaya J. (2017). Caracterización química del material particulado atmosférico del centro urbano de Huancayo, Perú. Rev. Soc. Quím Perú 83 (2), 187-199.

Tapia V., Carbajal L., Vásquez V., Espinoza R., Vásquez-Velasquez C., Steenland K. y Gonzales G.F. (2018). Reordenamiento vehicular y contaminación ambiental por material particulado ( 2.5 y 10$)$, dióxido de azufre y dióxido de nitrógeno en Lima Metropolitana, Perú. Rev. Peru. Med. Exp. Salud Pública 35 (2), 190-197. https://doi.org/10.17843/ rpmesp.2018.352.3250.190

Thurston G.D. y Spengler J.D. (1985). A quantitative assessment of source contributions to inhalable particulate matter pollution in Metropolitan Boston. Atmos. Environ. 19 (1), 9-25. https://doi.org/10.1016/00046981(85)90132-5

Viana M., Kuhlbusch T.A.J., Querol X., Alastuey A., Harrison R.M., Hopke P.K., Winiwarter W., Vallius M., Szidat S., Prévôt A.S.H., Hueglin C., Bloemen H., Wåhlin P., Vecchi R., Miranda A.I., Kasper-Giebl A., Maenhaut W. y Hitzenberger R. (2008). Source apportionment of particulate matter in Europe: A review of methods and results. J. Aerosol Sci. 39 (10), 827-849. https://doi.org/10.1016/j.jaerosci.2008.05.007

Wedepohl K H. (1995). The composition of the continental crust. Geochim. Cosmochim. Acta 59 (7), 1217-1232. https://doi.org/10.1016/0016-7037(95)00038-2 